\title{
Light-Matter Response in Nonrelativistic Quantum Electrodynamics
}

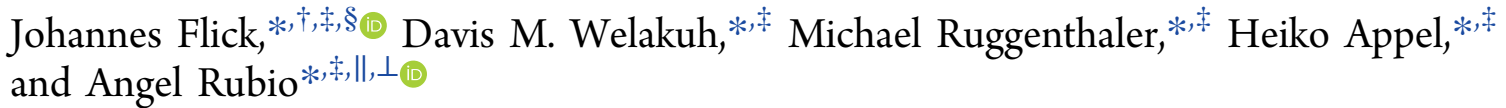

$\dagger$ John A. Paulson School of Engineering and Applied Sciences, Harvard University, Cambridge, Massachusetts 02138, United States ${ }^{\ddagger}$ Max Planck Institute for the Structure and Dynamics of Matter and Center for Free-Electron Laser Science and Department of Physics, Luruper Chaussee 149, 22761 Hamburg, Germany

"Center for Computational Quantum Physics, Flatiron Institute, 162 Fifth Avenue, New York, New York 10010, United States

${ }^{\perp}$ Nano-Bio Spectroscopy Group, Departamento de Fisica de Materiales, Universidad del País Vasco UPV/EHU- 20018 San

Sebastián, Spain

\section{Supporting Information}

ABSTRACT: We derive the full linear-response theory for nonrelativistic quantum electrodynamics in the long wavelength limit and provide a practical framework to solve the resulting equations by using quantum-electrodynamical density-functional theory. We highlight how the coupling between quantized light and matter changes the usual response functions and introduces cross-correlated lightmatter response functions. These cross-correlation responses lead to measurable changes in Maxwell's equations due to the quantum-matter-mediated photon-photon interactions. Key features of treating the combined matter-photon response are
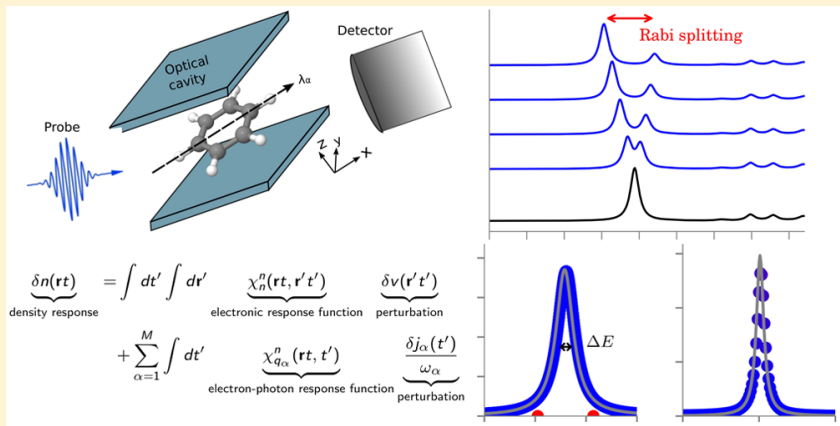
that natural lifetimes of excitations become directly accessible from first-principles, changes in the electronic structure due to strong light-matter coupling are treated fully nonperturbatively, and self-consistent solutions of the back-reaction of matter onto the photon vacuum and vice versa are accounted for. By introducing a straightforward extension of the random-phase approximation for the coupled matter-photon problem, we calculate the ab initio spectra for a real molecular system that is coupled to the quantized electromagnetic field. Our approach can be solved numerically very efficiently. The presented framework leads to a shift in paradigm by highlighting how electronically excited states arise as a modification of the photon field and that experimentally observed effects are always due to a complex interplay between light and matter. At the same time the findings provide a route to analyze as well as propose experiments at the interface between quantum chemistry, nanoplasmonics and quantum optics.

KEYWORDS: strong light-matter coupling, quantum-electrodynamical density functional theory, benzene molecule, linear-response theory, excited states

$\mathrm{R}$ ecent years have seen tremendous experimental advances in the nascent field of strongly coupled light-matter systems. ${ }^{1,2}$ In particular, new experimental advances have been demonstrated in polaritonic chemistry, ${ }^{3-5}$ solid-state physics, ${ }^{6}$ biological systems, ${ }^{7}$ nanoplasmonics, ${ }^{8,9}$ two-dimensional materials, ${ }^{10,11}$ or optical waveguides, ${ }^{12}$ among others.

In this so-called strong-coupling regime, as a result of mixing matter and photon degrees-of-freedom, ${ }^{13,14}$ novel effects emerge such as changes in chemical pathways ${ }^{15-17}$ groundstate electroluminescence, ${ }^{18}$ cavity-controlled chemistry for molecular ensembles, ${ }^{19,20}$ or optomechanical coupling in optical cavities, ${ }^{21}$ new topological phases of matter, ${ }^{22}$ superradiance, $^{23}$ or superconductivity. ${ }^{24}$

Due to the inherent complexity of such coupled fermionboson problems described in general by quantum electrodynamics (QED), the theoretical treatment is usually drastically simplified. One common approximation is to restrict the description of the system to simplified effective models that heavily rely on input parameters. Current state of the art in the theoretical description of strong light-matter coupling very often employs a few-level approximation. This approximation leading to the Rabi or Jaynes-Cummings model ${ }^{25,26}$ in the single-emitter case, or the Dicke model ${ }^{27}$ in the many-emitter case, is however often not sufficient, ${ }^{28,29}$ in particular, when observables besides the energy are of interest, ${ }^{29}$ such as in experimental setups involving the modification of chemical reactivity. ${ }^{1}$

Alternatively, in linear spectroscopy, the current theoretical description is built on the semiclassical approximation. ${ }^{30}$ Herein, the many-particle electronic system is treated quantum mechanically and the electromagnetic field appears as an external perturbation. As an external perturbation, the

Received: May 28, 2019

Published: October 2, 2019 
electromagnetic field probes the quantum system, but is not a dynamical variable of the complete system (see also Supporting Information, S1). Since in the strong-coupling regime light and matter must be on the same level, a semiclassical approximation is not adequate, and the feedback between light and matter has to be considered.

It is, however, long known that the radiative lifetimes are finite. Furthermore, experimentally excited-state properties are usually inferred from (de)excitations of the photon field, which is in stark contrast to the usual semiclassical theoretical description based solely on the electronic subsystem.

In free-space, this mismatch can be circumvented since excited-state properties such as radiative lifetimes of atoms and molecules can be calculated perturbatively using the theory of Wigner-Weisskopf, ${ }^{31}$ employing the Markov approximation. However, this perturbative treatment of the coupling of light and matter becomes insufficient in the case that strong lightmatter coupling is achieved, for example, due to many emitters or due to reducing the mode volume of a cavity. In such cases, the Markov approximation breaks down and the WignerWeisskopf theory is not applicable anymore. ${ }^{32}$ Additionally, it is not straightforward how to extend the original formulation of Wigner-Weisskopf to many electronic levels and, hence, to an $\mathrm{ab}$ initio treatment of electronic systems.

As a consequence, the current literature shows a large gap for situations, where light and matter is strongly coupled and observables such as excited-state densities, radiative lifetimes, or electron-photon correlated observables of interest. A good example is the control of the radiative lifetimes of single molecules ${ }^{33,34}$ by changing the environment. In such cases, the properties of the many-body system are changed, for example, the excitation energies and lifetimes are strongly modified. This happens because certain modes of the photon vacuum field are enhanced which can lead to a strong coupling of light with matter. Alternatively, increasing the number of particles leads to an enhancement of the coupling due to the self-consistent back-reaction of matter onto the photon field and vice versa. It is important to realize that such changes are nonperturbative for the photon field as well as for the matter subsystem and hence need a self-consistent implementation. This fact is most pronounced in the appearance of polaritonic states and their influence on chemical and physical properties of matter. ${ }^{1,13}$

In this paper, we close this gap by presenting a practical and general framework that subsumes electronic-structure theory, nanoplasmonics, and quantum optics. We present a description that challenges our conception of light and matter as distinct entities $^{35}$ and that expresses the excited states as modifications of the photon field. We do so by introducing a linear-response formalism for coupled matter-photon systems. This formalism leads naturally to the ability to calculate radiative lifetimes in arbitrary photon environments, including free-space, high- $Q$ optical cavity or nanoplasmonic structures. We make this approach practical by introducing a linear-response framework for quantum-electrodynamical density-functional theory (QEDFT). ${ }^{13,14,36-38}$ This development is specifically timely since QEDFT has now been successfully applied to real systems in equilibrium, ${ }^{39}$ which demonstrates the feasibility of $\mathrm{ab}$ initio strong-coupling calculations, yet an accurate and efficient approach to excited states within QEDFT has been missing. This work therefore furthermore closes a gap within the QEDFT framework. We further want to note that, there have been different studies in literature that are devoted to including the classical feedback of the light field to the matter systems all in dipole approximation, such as for specific systems ${ }^{40,41}$ or reduced dimensionality. ${ }^{42}$ The presented work not only generalizes these approaches, but also provides a clear path to how to include the quantum effects of the light field for this feedback.

\section{LIGHT-MATTER INTERACTION IN THE LONG WAVELENGTH LIMIT}

Our fundamental description of how the charged constituents of atoms, molecules, and solid-state systems, that is, electrons and positively charged nuclei, interact is based on QED; ${ }^{13,43-45}$ thus, the interaction is mediated via the exchange of photons. Adopting the Coulomb gauge for the photon field allows us to single out the longitudinal interaction among the particles, which gives rise to the well-known Coulomb interaction and leaves the photon field purely transversal. Assuming then that the kinetic energies of the nuclei and electrons are relatively small allows us to take the nonrelativistic limit for the matter subsystem of the coupled photon-matter Hamiltonian, which gives rise to the so-called Pauli-Fierz Hamiltonian ${ }^{13,37,45}$ of nonrelativistic QED. In a next step, one then usually assumes that the combined matterphoton system is in its ground state such that the transversal charge currents are small and that the coupling to the (transversal) photon field is very weak. Besides the Coulomb interaction, it is then only the physical mass of the charged constituents (bare plus electromagnetic mass ${ }^{45}$ ) that is a reminder of the photon field in the usual many-body Schrödinger Hamiltonian. In this work, however, we will not disregard the transversal photon field, which makes the presented framework much more versatile and applicable to situations of quantum mechanics and quantum optics at the same time (see also Appendix section, Photonic Observables and Radiative Lifetimes).

1.1. Spectroscopy from Quantum Description of Light-Matter Interaction. In the following, we consider cases in which the semiclassical approximation breaks down, as outlined in the introduction. In principle, QEDFT can be formulated for each level of theory of QED as presented in ref. ${ }^{37}$ As a consequence, the formalism outlined in this paper can be straightforwardly extended to more general formulations, including full minimal coupling beyond the dipole approximation $^{46}$ (in dipole approximation, only energy can be transferred between charged particles and the light field, but not momentum; thus, the dipole approximation is insufficient to describe processes such as the radiation reaction). In this manuscript, to illustrate the concepts, we restrict the discussion in the following to the dipole approximation and the lengthgauge.

To this end, from the Pauli-Fierz Hamiltonian, we make the long-wavelength or dipole approximation in the length-gauge $e^{47}$ since the wavelength of the photon modes are usually much larger than the extent of the electronic subsystem, as well as the Born-Oppenheimer approximations for the nuclei (the inclusion of the nuclei is straightforward; ${ }^{48}$ however, the presented formulation is perfectly suited to provide the photon-dressed modified potential-energy surfaces for the nuclei and, hence, access to modifications of chemical reactions in, e.g., optical cavities ${ }^{15,49}$ ), which leads (in SI units) to ${ }^{36,37,50}$ 


$$
\begin{aligned}
\hat{H}(t)= & \hat{H}_{\mathrm{e}}(t)+\sum_{\alpha=1}^{M} \frac{1}{2}\left[\hat{p}_{\alpha}^{2}+\omega_{\alpha}^{2}\left(\hat{q}_{\alpha}-\frac{\lambda_{\alpha}}{\omega_{\alpha}} \cdot \mathbf{R}\right)^{2}\right] \\
& +\frac{j_{\alpha}(t)}{\omega_{\alpha}} \hat{q}_{\alpha}
\end{aligned}
$$

where $\hat{H}_{\mathrm{e}}(t)$ is the standard many-body electronic Hamiltonian. ${ }^{51}$ We further restrict ourselves to arbitrarily many but a finite number $M$ of modes $\alpha \equiv(\mathbf{k}, s)$, with $s$ being the two transversal polarization directions that are perpendicular to the direction of propagation $\mathbf{k}$. The frequency $\omega_{\alpha}$ and polarization $\boldsymbol{\epsilon}_{\alpha}$ that enter in $\lambda_{\alpha}=\boldsymbol{\epsilon}_{\alpha} \lambda_{\alpha}$, with $\lambda_{\alpha}=S_{\mathbf{k}}(\mathbf{r}) / \sqrt{\epsilon_{0}}$ and mode function $S_{\mathbf{k}}(\mathbf{r})^{37}$ define these electromagnetic modes. $S_{\mathbf{k}}(\mathbf{r})$ is normalized, has the unit $1 / \sqrt{V}$ with the volume $V$, and we choose a reference point $\mathbf{r}_{0}$, where we have placed the matter subsystem to determine the fundamental coupling strength (all results presented in this paper are independent of $\mathbf{r}_{0}$ ). These photon modes couple via the displacement coordinate $\hat{q}_{\alpha}=\sqrt{\frac{\hbar}{2 \omega_{\alpha}}}\left(\hat{a}_{\alpha}+\hat{a}_{\alpha}^{\dagger}\right)$, where $\hat{q}_{\alpha}$ is given in terms of photon annihilation $\hat{a}_{\alpha}$ and creation $\hat{a}_{\alpha}^{\dagger}$ operators, to the total dipole moment $\mathbf{R}=\sum_{i=1}^{N} e \mathbf{r}_{i}$ (throughout this paper, we use the implicit definition $e=-|e|)$. The $\hat{q}_{\alpha}$ appears in the contribution of mode $\alpha$ to the displacement field $\hat{\mathbf{D}}_{\alpha}=\epsilon_{0} \omega_{\alpha} \lambda_{\alpha} \hat{q}_{\alpha \cdot}{ }^{47}$ Further, the conjugate momentum of the displacement coordinate is given by $\hat{p}_{\alpha}=-i \sqrt{\frac{\hbar \omega_{\alpha}}{2}}\left(\hat{a}_{\alpha}-\hat{a}_{\alpha}^{\dagger}\right)$. Besides a time-dependent external potential $v(\mathbf{r}, t)$, we also have an external perturbation $j_{\alpha}(t)$ that acts directly on the mode $\alpha$ of the photon subsystem. Here, $j_{\alpha}(t)$ is connected to a classical external charge current $\mathrm{J}(\mathbf{r}, t)$ that acts as a source for the inhomogeneous Maxwell's equation. Formally, however, due to the length-gauge transformations, the $j_{\alpha}(t)$ corresponds to the time-derivative of this (mode-resolved) classical external charge current ${ }^{36,37}$ (see also Appendix section, Self-Consistency of the Maxwell's Equation). Physically the static part $j_{\alpha, 0}$ merely polarizes the vacuum of the photon field and leads to a static electric field. ${ }^{38,52}$ The time-dependent part $\delta j_{\alpha}(t)$ then generates real photons in the mode $\alpha$. This term is also known as a source term in quantum field theory, ${ }^{43}$ where it generates the particles (here the photons) that are studied. From this perspective, it becomes obvious that instead of using $\delta j_{\alpha}(t)$ one could equivalently slightly change the initial state of the fully coupled system by adding incoming photons that then scatter off the coupled light-matter ground state. ${ }^{45}$

1.2. Linear Response in the Length Gauge. With the Hamiltonian of eq 1 in length gauge we can then in principle solve the corresponding time-dependent Schrödinger equation (TDSE) for a given initial state of the coupled matter-photon system $\Psi_{0}\left(\mathbf{r}_{1} \sigma_{1}, \ldots, \mathbf{r}_{N} \sigma_{N}, q_{1}, \ldots, q_{M}\right)$

$$
\mathrm{i} \hbar \frac{\partial}{\partial t} \Psi\left(\mathbf{r}_{1} \sigma_{1}, \ldots, t\right)=\hat{H}(t) \Psi\left(\mathbf{r}_{1} \sigma_{1}, \ldots, t\right)
$$

where $\sigma$ corresponds to the spin degrees of freedom. However, instead of trying to solve for the infeasible time-dependent many-body wave function, we restrict ourselves to weak perturbations $\delta v(\mathbf{r}, t)$ and $\delta j_{\alpha}(t)$ and assume that our system is in the ground state of the coupled matter-photon system initial time (in principle, also other initial states, e.g., an uncorrelated matter-photon state could be chosen). In this case, a first-order time-dependent perturbation theory can be used to approximate the dynamics of the coupled matter- photon system (for details, see Supporting Information, S2). This framework gives us access to linear spectroscopy, for example, the absorption spectrum of a molecule. Traditionally, if we made a decoupling of light and matter, that is, we assumed $\Psi_{0}\left(\mathbf{r}_{1} \sigma_{1}, \ldots, \mathbf{r}_{N} \sigma_{N}, q_{1}, \ldots, q_{M}\right) \simeq \psi_{0}\left(\mathbf{r}_{1} \sigma_{1}, \ldots, \mathbf{r}_{N} \sigma_{N}\right) \otimes$ $\varphi_{0}\left(q_{1}, \ldots, q_{M}\right)$, we would only consider the matter subsystem $\psi$ (the photonic part $\varphi$ would be completely disregarded). Physically, we would investigate the classical dipole field that the electrons induced due to a classical external perturbation $\delta v(\mathbf{r}, t)$. To determine this induced dipole field we would only consider the linear response of the density operator $\hat{n}(\mathbf{r})=$ $\sum_{i=1}^{N} \delta\left(\mathbf{r}-\mathbf{r}_{i}\right)$, which would be given by the usual densitydensity response function in terms of the electronic wave function $\psi_{0}$ only. In the following, we suppress the spin component of the wave function and focus exclusively on the spatial and mode dependence, i.e., $\Psi\left(\mathbf{r}_{1}, \ldots, \mathbf{r}_{N}, q_{1}, \ldots, q_{M} ; t\right)$.

In this work, however, since we do not assume the decoupling of light and matter, the full density-density response is taken with respect to the combined ground-state wave function $\Psi_{0}$ and is consequently different to the traditional density-density response. Further, since we can also perturb the photon field in the cavity by $\delta j_{\alpha}(t)$, which will subsequently induce density fluctuations, the density response $\delta n$ gets a further contribution leading to

$$
\delta n(\mathbf{r} t)=\int d t^{\prime} \int \mathrm{d} \mathbf{r}^{\prime} \chi_{n}^{n}\left(\mathbf{r} t, \mathbf{r}^{\prime} t^{\prime}\right) \delta v\left(\mathbf{r}^{\prime} t^{\prime}\right)+\sum_{\alpha=1}^{M} \int \mathrm{d} t^{\prime} \chi_{q_{\alpha}}^{n}\left(\mathbf{r} t, t^{\prime}\right) \delta j_{\alpha}\left(t^{\prime}\right)
$$

Here the response function $\chi_{n}^{n}\left(\mathbf{r} t, \mathbf{r}^{\prime} t^{\prime}\right)$ corresponds to the density-density response but with respect to the coupled light-matter ground state and $\chi_{q_{\alpha}}^{n}\left(\mathbf{r} t, t^{\prime}\right)$ corresponds to the density response induced by changing the photon field. In the standard linear-response formulation, due to the decoupling ansatz, changes in the transversal photon field would not induce any changes in the electronic subsystem. Since obviously we now have a cross-talk between light and matter, we accordingly have also a genuine linear-response of the quantized light field

$$
\delta q_{\alpha}(t)=\int \mathrm{d} t^{\prime} \int \mathrm{d} \mathbf{r}^{\prime} \chi_{n}^{q_{\alpha}}\left(t, \mathbf{r}^{\prime} t^{\prime}\right) \delta v\left(\mathbf{r}^{\prime} t^{\prime}\right)+\sum_{\alpha^{\prime}=1}^{M} \int \mathrm{d} t^{\prime} \chi_{q_{\alpha^{\prime}}}^{q_{\alpha}}\left(t, t^{\prime}\right) \delta j_{\alpha^{\prime}}\left(t^{\prime}\right)
$$

where $\chi_{n}^{q_{\alpha}}\left(t, \mathbf{r}^{\prime} t^{\prime}\right)$ is the full response of the photons due to perturbing the electronic degrees, and $\chi_{q_{\alpha}{ }^{\prime}}^{q_{\alpha}}\left(t, t^{\prime}\right)$ is the photonphoton response function. For an alternative definition of $\delta q_{\alpha}(t)$, we also refer the reader to eq S14 in the Supporting Information. The response function $\chi_{n}^{q_{\alpha}}\left(t, \mathbf{r} t^{\prime}\right)$ is, in general, not trivially connected to $\chi_{q_{\alpha}}^{n}\left(\mathbf{r} t, t^{\prime}\right)$, due to the different timeordering of $t$ and $t^{\prime}$.

The entire linear-response in nonrelativistic QED for the density and photon coordinate can also be written in matrix form. ${ }^{53}$ In this form, we clearly see that the density response of the coupled matter-photon system depends on whether we use a classical field $\delta v(\mathbf{r}, t)$, photons, which are created by $\delta j_{\alpha}(t)$, or combinations thereof for the perturbation. Furthermore, we can also decide to not consider the classical response of the coupled matter-photon system due to $\delta n(\mathbf{r}, t)$, but rather directly monitor the quantized modes of the photon field $\delta q_{\alpha}(t)$. This response yet again depends on whether we choose to use a classical field $\delta v(\mathbf{r}, t)$ that induces photons in mode $\alpha$ or whether we directly generate those photons by an external current $\delta j_{\alpha}(t)$, and we also see that the different modes 

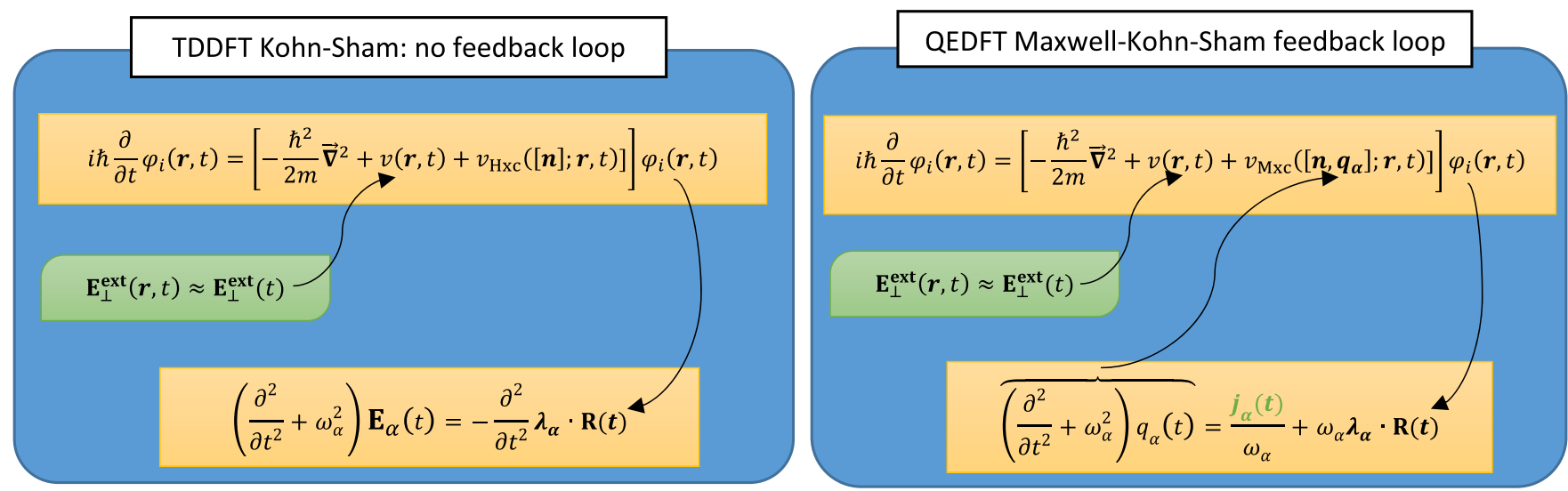

Figure 1. Schematics of the Maxwell KS approach contrasted with schematics of the usual semiclassical KS theory. While in the semiclassical approach the KS orbitals are used as fixed input into the mode-resolved inhomogeneous Maxwell's equation in vacuum through the total dipole $\mathbf{R}(t)=\int \operatorname{drer} \sum_{i}\left|\varphi_{i}(\mathbf{r}, t)\right|^{2}$ (see also Appendix section, Self-Consistency of the Maxwell's Equation), in the Maxwell KS framework the induced field acts back on the orbitals, which leads to an extra self-consistency cycle.

are coupled, that is, that photons interact. Similarly, as charged particles interact via coupling to photons, also photons interact via coupling to the charged particles. Keeping the coupling to the photon field explicitly therefore, on the one hand, changes the standard spectroscopic observables and, on the other hand, also allows for many more spectroscopic observables than in the standard matter-only theory.

1.3. Maxwell-Kohn-Sham Self-Consistent LinearResponse Theory. The problem with this general framework in practice is that already in the simplified matter-only theory, we usually cannot determine the exact response functions of a many-body system. The reason is that the many-body wave functions, which we use to define the response functions, are difficult, if not impossible, to determine beyond simple model systems. So, in practice, we need a different approach that avoids the many-body wave functions. Several approaches exist that employ reduced quantities instead of wave functions. ${ }^{54-56}$ The workhorse of these many-body methods is DFT and its time-dependent formulation TDDFT. ${ }^{57-59}$ Both theories have been extended to the general coupled matter-photon systems within the framework of QED. ${ }^{13,36-38,48}$

QEDFT allows us to solve instead of the TDSE equivalently a nonlinear fluid equation for the charge density $n(\mathbf{r}, t)$ coupled nonlinearly to the mode-resolved inhomogeneous Maxwell's equation. ${ }^{36-38,60}$ While these equations are, in principle, easy to handle numerically, we do not know the forms of all the different terms explicitly in terms of the basic variables of QEDFT, that is, $\left(n(\mathbf{r}, t), q_{\alpha}(t)\right)$. To find accurate approximations, one then employs the Kohn-Sham (KS) scheme, where we model the unknown terms by a numerically easy to handle auxiliary system in terms of wave functions. The simplest approach is to use noninteracting fermions and bosons that lead to a similar set of equations, which are however uncoupled. Enforcing that both give the same density and displacement field dynamics gives rise to mean-field exchange-correlation (Mxc) potentials and currents. ${ }^{52,61,62}$ Formally, this Mxc potential and current is defined as the difference of the potential/current that generate a prescribed internal pair in the auxiliary noninteracting and uncoupled system $\left(v_{s}([n], \mathbf{r}, t), j_{\alpha}^{\mathrm{s}}\left(\left[q_{\alpha}\right], t\right)\right)$; (the subscript "s" is usually not explained in the density-functional literature, but we can assume that it refers to "single particle", as the potential often appears in effective single-particle equations ${ }^{62}$ ) and the potential/current that generates the same pair in the physical system defined by eq 1 , which we denote by $\left(v\left(\left[n, q_{\alpha}\right], \mathbf{r}, t\right)\right.$, $\left.j_{\alpha}\left(\left[n, q_{\alpha}\right], t\right)\right)$, that is,

$$
\begin{aligned}
& v_{\mathrm{Mxc}}\left(\left[n, q_{\alpha}\right], \mathbf{r}, t\right)=v_{\mathrm{s}}([n], \mathbf{r}, t)-v\left(\left[n, q_{\alpha}\right], \mathbf{r}, t\right) \\
& j_{\alpha, \mathrm{M}}([n], t)=j_{\alpha}^{\mathrm{s}}\left(\left[q_{\alpha}\right], t\right)-j_{\alpha}\left(\left[n, q_{\alpha}\right], t\right)=-\omega_{\alpha}^{2} \lambda_{\alpha} \cdot \mathbf{R}(t)
\end{aligned}
$$

In the time-dependent case, we only have a mean-field contribution to the Mxc current, ${ }^{36,38}$ where the total dipole moment is written as $\mathbf{R}(t)=\int \operatorname{drer} n(\mathbf{r}, t)$. Further, we have ignored the so-called initial-state dependence because we assume (for notational simplicity and without loss of generality) in the following that we always start from a ground state $^{62,63}$ of the matter-photon coupled system. In this way, we can recast the coupled Maxwell-quantum-fluid equations in terms of coupled nonlinear Maxwell-KS equations for auxiliary electronic orbitals, which sum to the total density $\sum_{i}\left|\varphi_{i}(\mathbf{r}, t)\right|^{2}$ $=n(\mathbf{r}, t)$, and the displacement fields $q_{\alpha}(t)$, that is,

$$
\begin{aligned}
& i \hbar \frac{\partial}{\partial t} \varphi_{i}(\mathbf{r}, t)=\left[-\frac{\hbar^{2}}{2 m_{e}} \nabla^{2}+v_{\mathrm{KS}}\left(\left[v, n, q_{\alpha}\right], \mathbf{r}, t\right)\right] \varphi_{i}(\mathbf{r}, t) \\
& \left(\frac{\partial^{2}}{\partial t^{2}}+\omega_{\alpha}^{2}\right) q_{\alpha}(t)=-\frac{j_{\alpha}(t)}{\omega_{\alpha}}+\omega_{\alpha} \lambda_{\alpha} \cdot \mathbf{R}(t)
\end{aligned}
$$

Here we use the self-consistent KS potential $v_{\mathrm{KS}}\left(\left[v, n, q_{\alpha}\right], \mathbf{r}, t\right)$ $=v(\mathbf{r}, t)+v_{\mathrm{Mxc}}\left(\left[n, q_{\alpha}\right], \mathbf{r}, t\right)$ that needs to depend on the fixed physical potential $v(\mathbf{r}, t),{ }^{62}$ and instead of the full bosonic KS equation for the modes $\alpha$, we just provide the Heisenberg equation for the displacement field. Although the auxiliary bosonic wave functions might be useful for further approximations, it is only $q_{\alpha}(t)$ that is physically relevant, and thus, we get away with merely coupled classical harmonic oscillators, that is, the mode resolved inhomogeneous Maxwell's equation. To highlight the extra self-consistency due to coupling between light and matter, we contrast the traditional electron-only KS theory with the Maxwell KS theory in Figure 1. It is then useful to divide the Mxc potential into the usual Hartree-exchange-correlation ( $\mathrm{Hxc}$ ) potential that we know from electronic TDDFT and a correction term that we call photon-exchange-correlation potential ( $\mathrm{pxc}$ ), that is,

$$
v_{\mathrm{Mxc}}\left(\left[n, q_{\alpha}\right], \mathbf{r}, t\right)=v_{\mathrm{Hxc}}([n], \mathbf{r}, t)+v_{\mathrm{pxc}}\left(\left[n, q_{\alpha}\right], \mathbf{r}, t\right)
$$




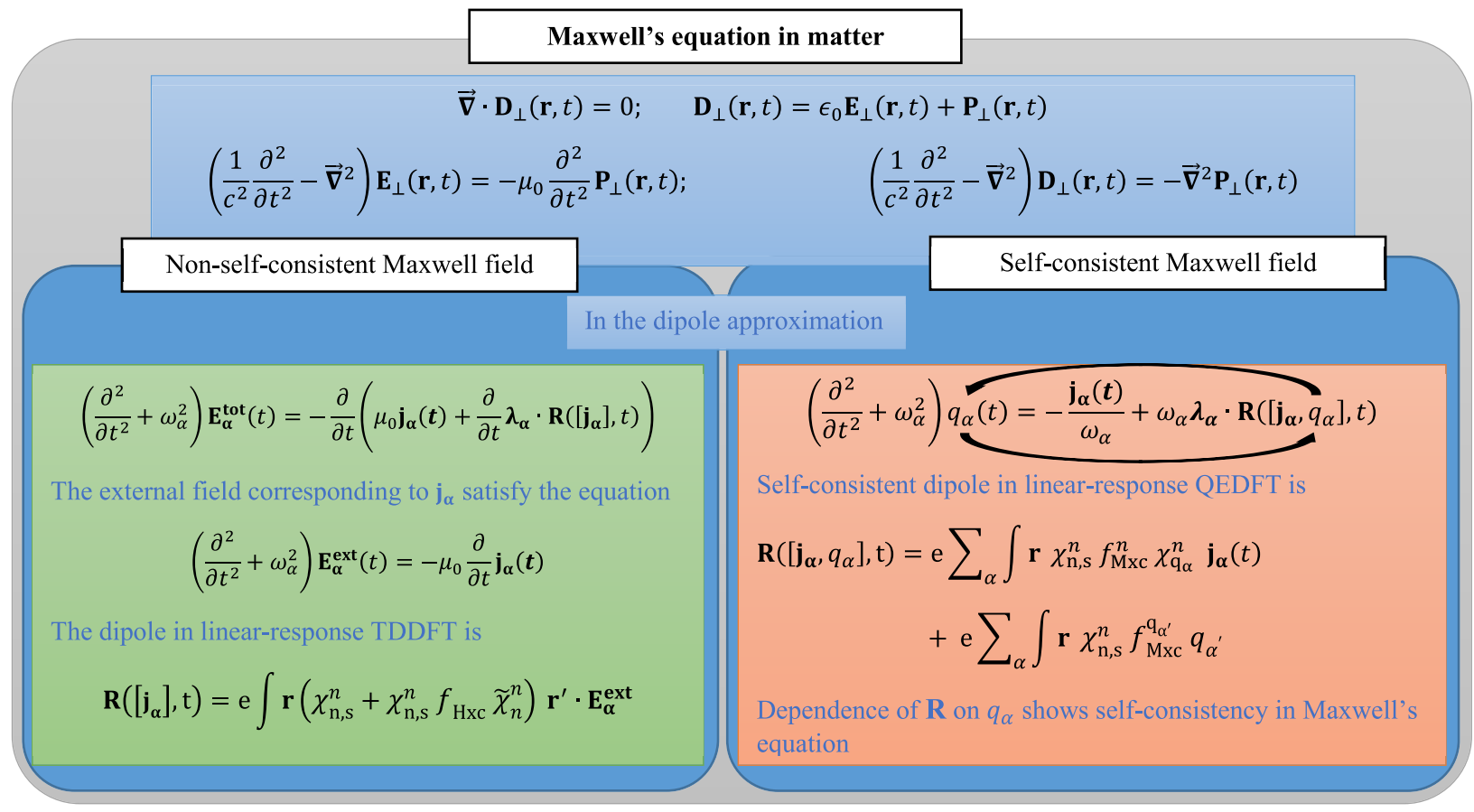

Figure 2. Schematics that contrasts the usual Maxwell's equation (left) with the fully self-consistent Maxwell's equation (right). Top: The induced transversal electric field $\mathbf{E}_{\perp}$ as a consequence of the induced polarization $\mathbf{P}_{\perp}$, which can be equivalently expressed in terms of the auxiliary displacement field $\mathbf{D}_{\perp}$. Left: mode-resolved nonself-consistent Maxwell's equation with no backreaction. The external charge current $\mathbf{j}_{\alpha}$ induces the external electric field in $\mathbf{E}_{\alpha}^{\text {tot }}=\mathbf{E}_{\alpha}+\mathbf{E}_{\alpha}^{\text {ext }}$, which acts as an external perturbation through the dipole. Since the constituents of $\tilde{\chi}_{n}^{n}$ expressed in TDDFT are purely electronic, the induced field does not couple back to the Maxwell field. Right: self-consistent Maxwell's equation in which $\mathbf{j}_{\alpha}$ induces the internal field $q_{\alpha}(t)$ through the electron-photon correlated dipole which has an explicit dependence as seen in the QEDFT form of $\chi_{q_{\alpha}}^{n}$. The selfconsistency of the induced field through the dipole introduces nonlinearities in the coupled system and, thus, changes the Maxwell field at the level of linear-response.

Clearly, the correction term $v_{\text {pxc }}$ will vanish if we take the coupling $\left|\lambda_{\alpha}\right|$ to zero and recover the purely electronic case. Since by construction the Maxwell KS system reproduces the exact dynamics, we also recover the exact linear-response of the interacting coupled system (see also Supporting Information, S3). We can express this with the help of the Mxc kernels defined by the functional derivatives of the Mxc quantities

$$
\begin{aligned}
& f_{\mathrm{Mxc}}^{n}\left(\mathbf{r} t, \mathbf{r}^{\prime} t^{\prime}\right)=\frac{\delta v_{\mathrm{Mxc}}(\mathbf{r} t)}{\delta n\left(\mathbf{r}^{\prime} t^{\prime}\right)}, f_{\mathrm{Mxc}}^{q_{\alpha}}\left(\mathbf{r} t, t^{\prime}\right)=\frac{\delta v_{\mathrm{Mxc}}(\mathbf{r} t)}{\delta q_{\alpha}\left(t^{\prime}\right)}, \\
& g_{\mathrm{M}}^{n_{\alpha}}\left(t, \mathbf{r}^{\prime} t^{\prime}\right)=\frac{\delta j_{\alpha, \mathrm{M}}(t)}{\delta n\left(\mathbf{r}^{\prime} t^{\prime}\right)}, g_{\mathrm{M}}^{q_{\alpha^{\prime}}}\left(t, t^{\prime}\right)=\frac{\delta j_{\alpha, \mathrm{M}}(t)}{\delta q_{\alpha^{\prime}}\left(t^{\prime}\right)} \equiv 0
\end{aligned}
$$

and use the corresponding definitions for the Hxc kernel (that only for the variation with respect to $n$ has a nonzero contribution) and the pxc kernels. We note that, using eq 6, we explicitly find

$$
g_{\mathrm{M}}^{n_{\alpha}}\left(t-t^{\prime}, \mathbf{r}\right)=-\delta\left(t-t^{\prime}\right) \omega_{\alpha}^{2} \lambda_{\alpha} \cdot e \mathbf{r}
$$

and $g_{\mathrm{M}}^{q_{\alpha \prime}}\left(t, t^{\prime}\right)$ vanishes, since $j_{\alpha, M}$ in eq 6 has no functional dependency on $q_{\alpha}$. Via these kernels, we find with $\chi_{n, s}^{n}\left(\mathbf{r} t, \mathbf{r}^{\prime} t^{\prime}\right)$ and $\chi_{q_{\alpha}^{\prime}, s}^{q_{\alpha}}\left(t, t^{\prime}\right)$, where $\chi_{q_{\alpha}, s}^{q_{\alpha}, s}\left(t, t^{\prime}\right) \equiv 0$ for $\alpha \neq \alpha^{\prime}$, the uncoupled and noninteracting response functions that

$$
\begin{aligned}
\chi_{n}^{n}\left(\mathbf{r} t, \mathbf{r}^{\prime} t^{\prime}\right)= & \chi_{n, s}^{n}\left(\mathbf{r} t, \mathbf{r}^{\prime} t^{\prime}\right)+\iint \mathrm{d} \mathbf{x} \mathrm{d} \tau \chi_{n, \mathrm{~s}}^{n}(\mathbf{r} t, \mathbf{x} \tau) \\
& \left(\iint \mathrm{d} \tau^{\prime} \mathrm{d} \mathbf{y} f_{\mathrm{Mxc}}^{n}\left(\mathbf{x} \tau, \mathbf{y} \tau^{\prime}\right) \chi_{n}^{n}\left(\mathbf{y} \tau^{\prime}, \mathbf{r}^{\prime} t^{\prime}\right)\right. \\
& \left.+\sum_{\alpha} \int \mathrm{d} \tau^{\prime} f_{\mathrm{Mxc}}^{q_{\alpha}}\left(\mathbf{x} \tau, \tau^{\prime}\right) \chi_{n}^{q_{\alpha}}\left(\tau^{\prime}, \mathbf{r}^{\prime} t^{\prime}\right)\right) \\
\chi_{q_{\alpha^{\prime}}}^{q_{\alpha}}\left(t, t^{\prime}\right)= & \chi_{q_{\alpha^{\prime}, s}^{q_{\alpha}}}^{q^{\prime}}\left(t, t^{\prime}\right)+\sum_{\beta} \iiint \mathrm{d} \tau \mathrm{d} \tau^{\prime} \mathrm{d} \mathbf{x} \chi_{q_{\beta, s}}^{q_{\alpha}}(t, \tau) g_{M}^{n_{\beta}}\left(\tau, \mathbf{x} \tau^{\prime}\right) \\
& \times \chi_{q_{\alpha^{\prime}}}^{n}\left(\mathbf{x} \tau^{\prime}, t^{\prime}\right)
\end{aligned}
$$

and accordingly for the mixed matter-photon response functions

$$
\begin{aligned}
\chi_{q_{\alpha}}^{n}\left(\mathbf{r} t, t^{\prime}\right)= & \iint \mathrm{d} \tau \mathrm{d} \mathbf{x} \chi_{n, s}^{n}(\mathbf{r} t, \mathbf{x} \tau)\left(\iint \mathrm{d} \tau^{\prime} \mathrm{d} \mathbf{y} f_{\mathrm{Mxc}}^{n}\left(\mathbf{x} \tau, \mathbf{y} \tau^{\prime}\right) \chi_{q_{\alpha}}^{n}\left(\mathbf{y} \tau^{\prime}, t^{\prime}\right)\right. \\
& \left.+\sum_{\alpha^{\prime}} \int \mathrm{d} \tau^{\prime} f_{\mathrm{Mxc}}^{q_{\alpha^{\prime}}}\left(\mathbf{x} \tau, \tau^{\prime}\right) \chi_{q_{\alpha}}^{q_{\alpha^{\prime}}}\left(\tau^{\prime}, t^{\prime}\right)\right) \\
\chi_{n}^{q_{\alpha}}\left(t, \mathbf{r}^{\prime} t^{\prime}\right)= & \sum_{\beta} \iiint \mathrm{d} \tau \mathrm{d} \tau^{\prime} \mathrm{d} \mathbf{y} \chi_{q_{\beta, s}}^{q_{\alpha}}(t, \tau) g_{M}^{n_{\beta}}\left(\tau, \mathbf{y} \tau^{\prime}\right) \chi_{n}^{n}\left(\mathbf{y} \tau^{\prime}, \mathbf{r}^{\prime} t^{\prime}\right)
\end{aligned}
$$

Here we employed the formal connection between response functions and functional derivatives $\chi_{n}^{n}\left(\mathbf{r} t, \mathbf{r}^{\prime} t^{\prime}\right)=\delta n(\mathbf{r}, t) / \delta v\left(\mathbf{r}^{\prime}\right.$, $\left.t^{\prime}\right)$, as well as $\chi_{q_{\alpha}{ }^{\prime}}^{q_{\alpha}}\left(t, t^{\prime}\right)=\delta q_{\alpha}(t) / \delta j_{\alpha}{ }^{\prime}\left(t^{\prime}\right)$ and accordingly for the auxiliary system. The Mxc kernels correct the unphysical responses of the auxiliary system to match the linear response of the interacting and coupled problem. So, in practice, instead of the full wave function, what we need are approximations to the unknown Mxc kernels. Later we will provide such 
approximations, show how accurate they perform for a model system and then apply them to real systems. If we decouple light and matter, that is, $\Psi_{0} \simeq \psi_{0} \otimes \varphi_{0}$, and disregard the photon part $\varphi_{0}$ (as is usually done in many-body physics), we recover the response function of eq 10 with $f_{\mathrm{Mxc}}^{q_{\alpha}} \equiv 0$, and $f_{\mathrm{Mxc}}^{n}$ $\rightarrow f_{\mathrm{Hxc}}^{n}$. The response function, which is calculated with the bare matter initial state $\psi_{0}$, then obeys the usual Dyson-type equation relating the noninteracting and interacting response in $\operatorname{TDDFT}^{64,65}$ with $v_{\mathrm{Mxc}}\left(\left[n, q_{\alpha}\right], \mathbf{r}, t\right) \rightarrow v_{\mathrm{Hxc}}([n], \mathbf{r}, t)$.

1.4. Excited States as Properties of the Photon Field. Following the above discussion, the usual response functions will change and response functions are introduced if we keep the matter-photon coupling explicitly. This leads to many exciting consequences. First, we get the completely selfconsistent response of the system including all screening, retardation (we note that retardation processes which require the exchange of more than one photon are independent of a dipolar approximation) and other effects that become important when either the matter subsystem is becoming large ${ }^{66-69}$ or when strong-coupling situations are considered. Since light and matter influence each other nonperturbatively the usual simplified approximations that only treat one part of the system accurately become unreliable ${ }^{28,29}$ (see also the discussion in section 3.3). Second, due to the matter-mediated photon-photon interactions (see Appendix section, SelfConsistency of the Maxwell's Equation, and Figure 2), the Maxwell's equations become self-consistent. A very interesting consequence is that, in contrast to a purely classical theory, we can theoretically distinguish whether a system is perturbed by a free current (that, in turn, would generate a classical electromagnetic field) or by a free electromagnetic field, for example, a classical laser pulse. Third, the inclusion of the photon modes introduces the missing photon bath that leads to finite lifetimes (see Appendix section, Photonic Observables and Radiative Lifetimes, and section 3.2). In connection to this it becomes important that we suddenly have access to a wealth of observables that describe the photon field. Most importantly, this implies the possibility to completely change our perspective of excited states of atoms and molecules. Indeed, in line with the experimental situation where changes in the photon field give us information on the excited states, we can view excited-state properties as arising from quantum modifications of the Maxwell's equations in matter

$$
\left(\frac{\partial^{2}}{\partial t^{2}}+\omega_{\alpha}^{2}\right) \delta q_{\alpha}(t)=-\frac{\delta j_{\alpha}(t)}{\omega_{\alpha}}+\omega_{\alpha} \lambda_{\alpha} \cdot \int \mathrm{d} \mathbf{r e r} \delta n(\mathbf{r}, t)
$$

The response of the density is then found with help of the response functions eqs $10-13$. In the usual case of an external classical field $\delta v(\mathbf{r}, t)$ and $\delta j_{\alpha}(t)=0$, we then find the induced field by (suppressing detailed dependencies with $\int \mathrm{d} \mathbf{r} \rightarrow \int$ and $\left.\int \mathrm{d} \mathbf{r} \sum_{\alpha} \rightarrow f\right)$

$$
\begin{gathered}
\left(\frac{\partial^{2}}{\partial t^{2}}+\omega_{\alpha}^{2}\right) \delta q_{\alpha}(t)=\omega_{\alpha} \boldsymbol{\lambda}_{\alpha} \cdot \int e \mathbf{r} \chi_{n, \mathrm{~s}}^{n} \delta v+\omega_{\alpha} \boldsymbol{\lambda}_{\alpha} \\
\int e \mathbf{r} \chi_{n, \mathrm{~s}}^{n} f_{\mathrm{Mxc}}^{n} \chi_{n}^{n} \delta v+\omega_{\alpha} \boldsymbol{\lambda}_{\alpha} \cdot \bigvee e \mathbf{r} \chi_{n, \mathrm{~s}}^{n} f_{\mathrm{Mxc}}^{q_{\alpha^{\prime}}} \delta q_{\alpha^{\prime}}
\end{gathered}
$$

Here, the first term on the right-hand side corresponds to the noninteracting matter-response. However, due to the electron-electron interaction, we need to take into account also the self-polarization of interacting matter (second term).
Finally, the third term describes the matter-mediated photonphoton response. The excited states of the coupled lightmatter system are in this description changes in the photon field. That this perspective is actually quite natural becomes apparent if one considers the nature of the emerging resonances for a real system (see Figure 7). These resonances are mainly photonic in nature, as they describe the emission/ absorption of photons (see Appendix section, Photonic Observables and Radiative Lifetimes). Let us consider now in more detail what the terms on the right-hand side of the modified Maxwell's equations mean physically. First of all, in a matter-only theory the self-consistent solution of the Maxwell's equations together with the response of the bare matter-system would correspond approximately to the first two terms on the right-hand side (see Appendix section, Self-Consistency of the Maxwell's Equation). The photon-photon interaction would not be captured in such an approximate approach. Second, to highlight the physical content of the different terms, we can make the mean-field contributions due to

$$
v_{\mathrm{M}}(\mathbf{r} t)=\sum_{\alpha}\left(\int \mathrm{d} \mathbf{r}^{\prime} \lambda_{\alpha} \cdot e \mathbf{r}^{\prime} n\left(\mathbf{r}^{\prime} t\right)-\omega_{\alpha} q_{\alpha}(t)\right) \lambda_{\alpha} \cdot e \mathbf{r}+\int \mathrm{d} \mathbf{r}^{\prime} \frac{e^{2} n\left(\mathbf{r}^{\prime} t\right)}{4 \pi \epsilon_{0}\left|\mathbf{r}-\mathbf{r}^{\prime}\right|}
$$

explicit

$$
\begin{aligned}
& \left(\frac{\partial^{2}}{\partial t^{2}}+\omega_{\alpha}^{2}\right) \delta q_{\alpha}(t)=\omega_{\alpha} \boldsymbol{\lambda}_{\alpha} \cdot \int e \mathbf{r} \chi_{n, \mathrm{~s}}^{n} \delta v+\omega_{\alpha} \boldsymbol{\lambda}_{\alpha} \\
& \int e \mathbf{r} \chi_{n, \mathbf{s}}^{n}\left[\frac{e^{2}}{4 \pi \epsilon_{0}\left|\mathbf{r}^{\prime}-\mathbf{r}^{\prime \prime}\right|}+\sum_{\alpha^{\prime}}\left(\boldsymbol{\lambda}_{\alpha^{\prime}} \cdot e \mathbf{r}^{\prime \prime}\right) \boldsymbol{\lambda}_{\alpha^{\prime}} \cdot e \mathbf{r}^{\prime}\right] \chi_{n}^{n} \delta v \\
& -\omega_{\alpha} \boldsymbol{\lambda}_{\alpha} \cdot y e \mathbf{r} \chi_{n, \mathrm{~s}}^{n}\left(\omega_{\alpha^{\prime}} \boldsymbol{\lambda}_{\alpha^{\prime}} \cdot e \mathbf{r}^{\prime}\right) \delta q_{\alpha^{\prime}}+\omega_{\alpha} \boldsymbol{\lambda}_{\alpha} . \\
& \int e \mathbf{r} \chi_{n, \mathrm{~s}}^{n} f_{\mathrm{xc}}^{n} \chi_{n}^{n} \delta v+\omega_{\alpha} \boldsymbol{\lambda}_{\alpha} \cdot \bigvee e \mathbf{r} \chi_{n, \mathrm{~s}}^{n} f_{\mathrm{xc}}^{q_{\alpha^{\prime}}} \delta q_{\alpha^{\prime}}
\end{aligned}
$$

The second term on the right-hand side then corresponds to the random-phase approximation (RPA) to the instantaneous matter-matter polarization. Here, a term that corresponds to the dipole self-energy induced by the coupling to the photons arises. The third term on the right-hand side is the RPA approximation to the dipole-dipole mediated photon interaction. To give these terms further physical meaning, note that in the usual perturbative derivation of the van der Waals interaction ${ }^{44}$ the first two terms would cancel and leave the photonic dipole-dipole interaction that gives rise to the $R^{-6}$ for small distances and the $R^{-7}$ for larger distances. The rest are exchange-correlation (xc) contributions that arise due to more complicated interactions among the electrons and photons. The last term effectively describe photon-photon interactions mediated by matter. In addition, we want to highlight that $\mathrm{xc}$ contributions are directly responsible for multiphoton effects, such as two-photon or three-photon processes (see Figure 4). If we only keep the mean-field contributions of the coupled problem, we will denote the resulting approximation in the following as photon RPA (pRPA) to distinguish it from the bare RPA of only the Coulomb interaction. We see how the Maxwell's equations in matter become self-consistent due to bound charges, that is, fields due to the polarization of matter. A new term, the photon-photon interaction, appears. For free charges, that is, due to an external charge current $\delta j_{\alpha}(t)$, we see similar changes. Clearly, if we had no coupling to matter, then there would be no induced density change and we just find the 


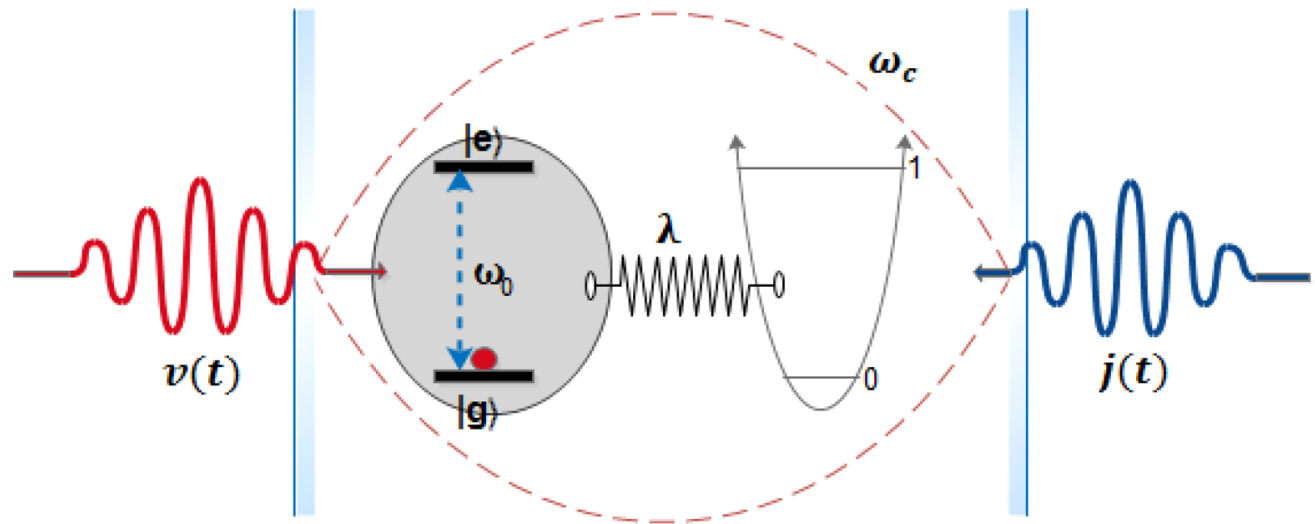

Figure 3. Two-level system (with excitation $\omega_{0}$ ) coupled to one mode of the radiation field (with frequency $\omega_{c}$ ). The matter subsystem is driven by an external classical field $v(t)$ and the photon mode is driven by an external classical current $j(t)$ and both subsystems are coupled with a coupling strength $\lambda$.
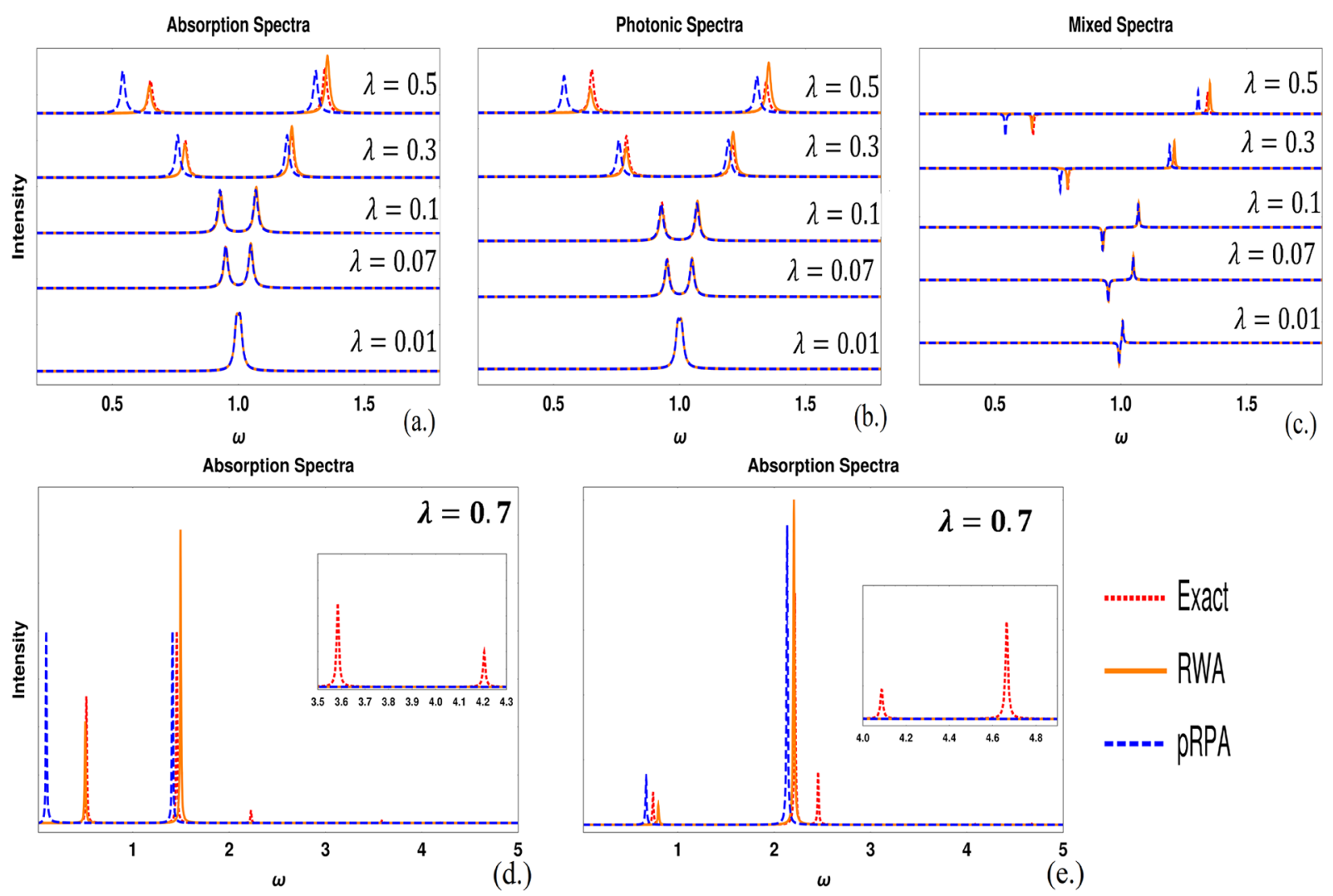

Figure 4. Linear-response spectra for the extended Rabi model (dotted-red) compared to the pRPA (dashed-blue) and RWA (full-orange) approximations and for different coupling strengths $\lambda$. (a) Absorption spectra due to matter-matter response, (b) spectra due to photon-photon response, (c) spectra due to matter-photon or photon-matter response. (d) The case for $\lambda=0.7$ shows all excitations that arise in strong coupling. $(\mathrm{a}-\mathrm{d})$ Resonant coupling. In (e), the field is halfway detuned from atomic resonance, that is, $\omega_{0}=2$ and $\omega_{\mathrm{c}}=1$ with strength and energies shifted to frequencies favoring 2-photon processes. The insets in $(\mathrm{d})$ and $(\mathrm{e})$ zoom into the frequency axis showing a many-photon process.

vacuum Maxwell's equations coupled to an external current for the electric field. In other terms, the displacement field trivially corresponds to the electric field (see Appendix section, SelfConsistency of the Maxwell's Equation).

\section{ILLUSTRATIVE EXAMPLES FOR THE COUPLED MATTER-PHOTON RESPONSE}

In this section, we discuss the perspective enabled by the linear response formalism of QEDFT in more detail for a simple and illustrative model system. We discuss a slight generalization of the Rabi model, ${ }^{70,71}$ which is the standard model of quantum optics. The Rabi model describes a single electron on two lattice sites/energy levels interacting with a single photon mode. We schematically depict the system in Figure 3 and present all further details of this system in Appendix section, Examples for the Coupled Matter-Photon Response: Details on the Rabi Model.

First, let us analyze the optical spectra for such a system and scrutinize the different approximations to the Mxc kernels. We will compare the numerical exact results, with the mean-field (pRPA) and the rotating-wave approximation (RWA). In 


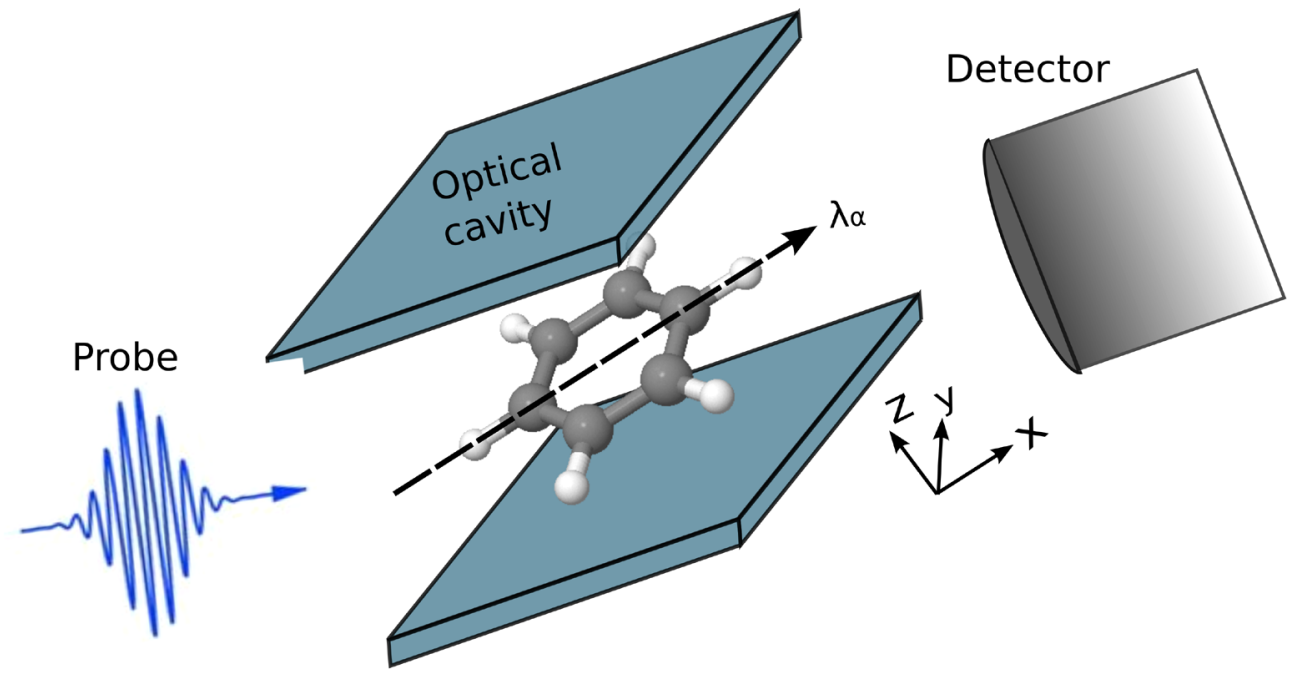

Figure 5. Schematic of absorption spectroscopy in optical cavities: Benzene $\left(\mathrm{C}_{6} \mathrm{H}_{6}\right)$ molecule and $\lambda_{\alpha}$ denotes the polarization direction of the photon field.

Figure $4 a-c$ we see how the optical spectra of the resonantly coupled system (i.e., $\delta=\omega_{0}-\omega_{\mathrm{c}}=0$ ) change for an increasing electron-photon coupling strength $\lambda$. Already for small coupling, the splitting of the electronic state into an upper and lower polariton becomes apparent. Approximately these states are given in terms of the RWA as $|+, 0\rangle$ and $|-, 0\rangle$. The difference in energy between the lower and upper polariton is called the Rabi splitting $\Omega_{\mathrm{R}}$ and is used to indicate the strength of the matter-photon coupling. In molecular experiments values of up to $\Omega_{\mathrm{R}} / \omega_{\mathrm{c}} \simeq 0.25$ have been measured. ${ }^{72,73} \mathrm{Up}$ to $\lambda$ $=0.1$ the different spectra for the exact (dotted-red), the pRPA (dashed-blue) as well as the RWA (full-orange) are in close agreement before they start to differ. Already the mean-field treatment is enough to recover the quantized matter-photon responses, even for the coupled matter-photon spectra in Figure 4c. Consequently, the pRPA seems a reasonable approximation for linear-response spectra even for relatively strong coupling situations. Only upon increasing the coupling strength further and thus going into the ultrastrong coupling regime, the discrepancies becomes large. For ultrastrong coupling (for $\lambda=0.3$ the Rabi splitting is already of the order of $0.5 \omega_{c}$ ), the approximations do not recover the exact results. Increasing further leads then to not only a disagreement in transition frequencies, but also the weights of the transitions become increasingly different.

Besides a simple check for the approximations to the Mxc kernels, the extended Rabi model also allows us to get some understanding of the response functions $\chi_{q}^{\sigma_{x}}, \chi_{\sigma_{x}}^{q}$, and $\chi_{q}^{q}$, where $\sigma_{x}$ is the expectation-value of the corresponding Pauli matrix and describes the density/occupation changes between the two sites/energy levels. This means we consider mixed spectroscopic observables, where we perturb one subsystem and then consider the response in the other. We analogously employ $\chi_{\sigma_{x}}^{q}(\omega)$ and $\chi_{q}^{\sigma_{x}}(\omega)$, respectively, to determine a "mixed polarizability” (see Supporting Information, S5). If we plot this mixed spectrum (see Figure $4 \mathrm{c}$ displayed in dotted-red for the numerically exact case), we find that we have positive and negative peaks. Indeed, this highlights that excitations due to external perturbations can be exchanged between subsystems, that is, energy absorbed in the electronic subsystem can excite the photonic subsystem and vice versa. The oscillator strength of the photonic spectrum (based on $\chi_{q}^{q}$ ) in Figure $4 \mathrm{~b}$ provides us with a measure of how strong the displacement field (and with this also the electric field) reacts to an external classical charge current with frequency $\omega$. Similarly, the mixed spectrum (based on $\chi_{q}^{\sigma_{x}}$ or $\chi_{\sigma_{x}}^{q}$ ) in Figure $4 \mathrm{c}$ provides us with information on how strong one subsystem of the coupled system reacts upon perturbing the other one. The oscillator strength here is not necessarily positive. What is absorbed by one subsystem can be transferred to the other.

In Figure 4d,e, we show specifically the absorption spectra of the Rabi model for ultrastrong coupling, that is, $\lambda=0.7$. In this regime, three new peaks arise for the exact case accounting for high-lying excited states with nonvanishing dipole moments due to the strong electron-photon coupling. The new absorption peaks in Figure $4 \mathrm{~d}$, also shown in the inset, describes the resonant coupling case which the RWA and pRPA fail to capture in strong coupling, since processes beyond one-photon are involved. Similarly, Figure $4 \mathrm{e}$ depicts the case where the field is half-detuned from the electronic resonance indicating a two-photon process. Clearly in ultrastrong coupling the absorption peaks are merely shifted close to the bare frequencies of the individual subsystems, but remain dressed by the photon field as new peaks arise due to the coupling. The pRPA and RWA capture the first of the two peaks around $\omega=2$, which is also the frequency of the atom, but fail to capture higher lying nonvanishing contributions to the spectra. These higher-lying peaks correspond to multiphoton processes. With more accurate approximation for the xc potential results closer to the exact ones can be obtained. We note at this point that the peaks in Figure 4 are artificially broadened and in reality correspond to sharp transitions due to excited states with infinite lifetimes. How to get lifetimes quantitatively will be discussed in the next section.

\section{COUPLED MATTER-PHOTON RESPONSE: REAL SYSTEMS}

In this section, we apply the introduced formalism in pRPA approximation to real systems. We make the linear-response formulation practical by reformulating the problem as an eigenvalue equation in the frequency-domain. For electrononly problems this formulation is known as the Casida 
equation. ${ }^{65}$ We refer the reader to Appendix section, LinearResponse Theory as a Pseudoeigenvalue Problem, for a derivation of our extension of the Casida equation, which includes transverse photon fields. For the following discussion, we consider benzene molecules in an optical cavity but the presented approach is not restricted to any specific system.

In Figure 5, we schematically depict the experimental setup for a photoabsorption experiment under strong light-matter coupling for a single molecule. First we study the prototypical cavity QED setup where a molecule is strongly coupled to a single cavity mode of a high- $Q$ cavity. In the second setup, we lift the restriction of only one mode and instead couple the benzene molecule to many modes that sample the electromagnetic vacuum field without enhancing the coupling to a specific mode by hand. In the third setup, we study the behavior of two molecules in an optical cavity, as well as a dissipative situation, where only a few modes are strongly coupled, embedded in a quasi-continuum of modes. In the last example, we analyze the strong coupling of a single molecule to a continuum of modes. We find a transition from Lorentzian line shape to a Fano line shape ${ }^{74}$ for increasing electronphoton coupling strength. These different setups provide us with an ab initio calculation for the spectrum of a real molecule in a high- $Q$ cavity, an ab initio determination of intrinsic lifetimes and an $a b$ initio calculation of the nonperturbative interplay between electronic structure, lifetime, and strongcoupling. The two last situations need a self-consistent treatment of photons and matter alike and cannot be captured by any available electronic-structure or quantum-optical method. All of those examples highlight the rich possibilities and perspectives that the QEDFT framework provides.

3.1. Strong Light-Matter Coupling. The first results we discuss are a set of calculations, where a benzene molecule is strongly coupled to a single photon mode in an optical high- $Q$ cavity (our approach could also describe strong light-matter coupling for other systems, e.g., nanoplasmonic systems ${ }^{8}$ and generalizations to quantum interactions in laser pulses could be done along the lines of ref 75). We have implemented the linear-response pseudoeigenvalue equation of eq 43 into the real-space code OCTOPUS ${ }^{76,77}$ and details of the numerical parameters are given in Appendix section, Numerical Details. The routines used to perform all calculations in this work will be made publicly available. They can be easily transported to any other first-principles code that has the matter linearresponse equations implemented to make them ready to describe the complete QED response, i.e., joint matterphoton response, as described in this work.

In the first calculation, we include a single cavity mode in resonance to the $\Pi-\Pi^{*}$ transition of the benzene molecule, ${ }^{76,78}$ that is, $\omega_{\alpha}=6.88 \mathrm{eV}$. For the light-matter coupling strength $\lambda_{\alpha}=\left|\lambda_{\alpha}\right|$, we choose five different values, that is, $\lambda_{\alpha}=(0,2.77,5.55,8.32,11.09) \mathrm{eV}^{1 / 2} / \mathrm{nm}$ that correspond to a transition from the weak to the strong-coupling limit and the cavity mode is assumed to be polarized along the $x$ direction.

Since in this manuscript, we focus on electron-photon coupling, we do not consider the coupling to the nuclei. Generalizations are straightforward, for example, along the line of ref 48. In experiment, in particular for molecular systems, the majority of the line-broadening is due to vibrational coupling, see, for example, refs 79 and 80 , for the optical spectra of benzene. Strong light-matter coupling for such systems will lead to the splitting of the peak into the lower and upper polariton and both peaks will inherit the vibrational line broadening of the electronic excitation outside the cavity, as has been shown in various experiments, for example, refs 81 and 82 .

In Figure 6, we show the absorption spectra for these different values of $\lambda_{\alpha}$. We start by discussing the $\lambda_{\alpha}=0$ case

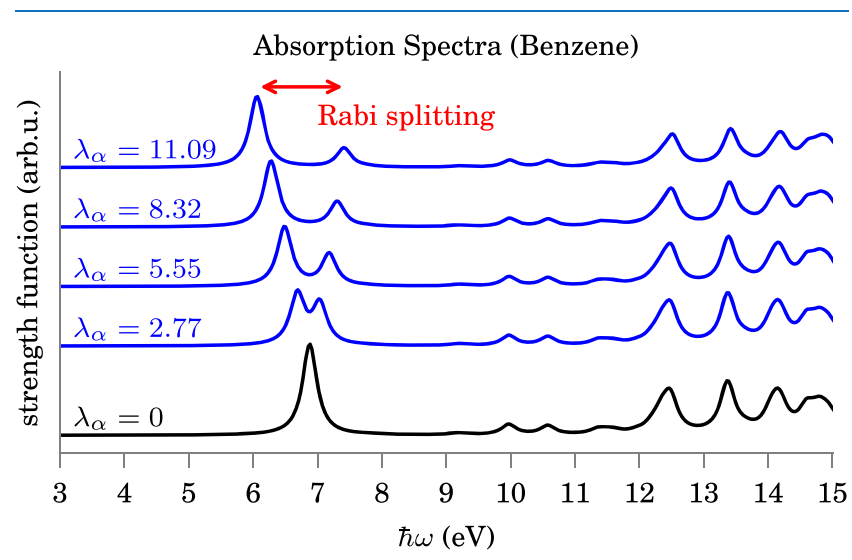

Figure 6. Absorption spectra for the benzene molecule in free space (black) and under strong light-matter coupling in an optical cavity to ultrastrong coupling (blue). The value for $\lambda_{\alpha}$ is given in units of $\left[\mathrm{eV}^{1 / 2} / \mathrm{nm}\right]$.

that is shown in black. This spectrum corresponds to a calculation of the benzene molecule in free space, and the spectrum is within the numerical capabilities identical to ref 76 . The spectrum in ref 76 has been obtained using an explicit time-propagation with finite time. In the limit of zero broadening and including all unoccupied states, we would find identical spectra with very long propagated spectra. We stress that here the broadening of the peaks is only done artificially since the photon bath is not included in the calculation. In the examples of sections 3.2 and 3.4 we include many modes and, hence, sample the photon bath nonperturbatively. We tune the electron-photon coupling strength $\lambda_{\alpha}$ in Figure 6. We find for increasing coupling strength a Rabi splitting of the $\Pi-\Pi^{*}$ peak into two polaritonic branches. The lower polaritonic branch has higher intensity, compared to the upper polaritonic peak. Numerical values for the excitation energy $E_{\mathrm{I}}$, the transition dipole moment $x_{\mathrm{I}}$ and the oscillator strength $f_{\mathrm{I}}$ are given in Table 1 in the Appendix. This demonstrates that ab initio theory is able to describe excited-state properties of strong light-matter coupling situations and captures the hybrid character of the

Table 1. Rabi Splitting of the $\Pi-\Pi^{*}$ Transition: ElectronPhoton Interaction Strength $\lambda_{\alpha}=\left|\lambda_{\alpha}\right|$, Excitation Energy $E_{I}$, Transition Dipole Moment $x_{I}$, and the Oscillator Strength $f_{I}$

$\begin{array}{cccc}\lambda_{\alpha}\left(\mathrm{eV}^{1 / 2} / \mathrm{nm}\right) & E_{I}(\mathrm{eV}) & \left\langle x_{I}\right\rangle(\mathrm{A}) & f_{I}(\text { a.u. }) \\ 0 & 6.88 & 0.952 & 0.546 \\ 2.77 & 6.69 & 0.721 & 0.304 \\ 2.77 & 7.03 & 0.626 & 0.241 \\ 5.55 & 6.49 & 0.791 & 0.355 \\ 5.55 & 7.18 & 0.550 & 0.190 \\ 8.32 & 6.28 & 0.848 & 0.395 \\ 8.32 & 7.30 & 0.482 & 0.149 \\ 11.09 & 6.06 & 0.896 & 0.426 \\ 11.09 & 7.41 & 0.420 & 0.114\end{array}$


combined matter-photon states. Thus, predictive theoretical first-principle calculations for excited-states properties of real systems strongly coupled to the quantized electromagnetic field are now available. This will allow unprecedented insights into coupled light-matter systems, since we have access to many observables that are not (or not well ${ }^{29}$ ) captured by quantum-optical models.

3.2. Lifetimes of Electronic Excitations from FirstPrinciples. Next, we consider how to obtain lifetimes from QEDFT linear-response theory. In this example, we explicitly couple the benzene molecule to a wide range of photon modes similar as in the spontaneous emission calculation of ref 83 While in ref 83 the system was simulated with 200 photon modes, we choose here now 80000 photon modes. The energies of the sampled photon modes cover densely a range from $0.19 \mathrm{meV}$, for the smallest energy up to $30.51 \mathrm{eV}$ for the largest one with a spacing of $\Delta \omega=0.38 \mathrm{meV}$. However, we do not sample the full three-dimensional mode space together with the two polarization possibilities per mode but rather consider a one-dimensional slice in mode space. This onedimensional sampling of mode frequencies will change the actual three-dimensional lifetimes, but for demonstrating the possibilities of obtaining lifetimes, this is sufficient (a detailed analysis of real lifetimes would include, besides a proper sampling of the mode space, considerations with respect to the bare mass of the particles). The sampling of the photon modes corresponds to the modes of a quasi-one-dimensional cavity. We choose a cavity of length $L_{x}^{83}$ in the $x$-direction, with a finite width in the other two directions that are much more confined. Thus, we employ $\omega_{\alpha}=\alpha c \pi / L_{x}$ and $\lambda_{\alpha}=\sqrt{\frac{2}{\hbar \epsilon_{0} L_{x} L_{y} L_{z}}} \sin \left(\omega_{\alpha} / c x_{0}\right) \mathbf{e}_{x}$, where $x_{0}=L_{x} / 2$ is the position of the molecule in the $x$-direction. While we have a sine mode function in the $x$-direction, we assume a constant mode function in the other directions. For this example, we choose a cavity of length $L_{x}=3250 \mu \mathrm{m}$ in the $x$-direction, $L_{y}=10.58 \AA$ in the $y$-direction, and $L_{z}=2.65 \AA$ in the $z$-direction.

The results of this calculation are shown in Figure 7. In Figure $7 \mathrm{a}$, we show the full spectrum. The electron-photon absorption function that has been obtained by coupling the benzene molecule to the quasi one-dimensional cavity with 80000 cavity modes is plotted in blue. Since we have sampled the photon part densely, we do not need to artificially broaden the peaks anymore. Formulated differently, we can directly plot the oscillator strength and the excitation energies of our resulting eigenvalue equation and do not need to employ the Lorentzian broadening anymore. In Figure 7, from blue (more photonic) to red (more electronic) for the electron-photon absorption spectrum we plot the different contributions of each pole in the response function. These results confirm our intuition that resonances are mainly photonic in nature and that a Maxwell's perspective of excited states is quite natural. In (b) we zoom to the $\Pi-\Pi^{*}$ transition. Due to quasi onedimensional nature of the quantization volume, we find a broadening of the peak that is larger than it is for the case of a three-dimensional cavity due to the sampling of the electromagnetic vacuum. This is similar to changing the vacuum of the electromagnetic field. Accordingly the lifetimes of the electronic states are shorter if the electromagnetic field is confined to one dimension and we will discuss this in the next section.

3.3. Connection to the Standard Wigner-Weisskopf Theory. If the coupling between light and matter is very weak

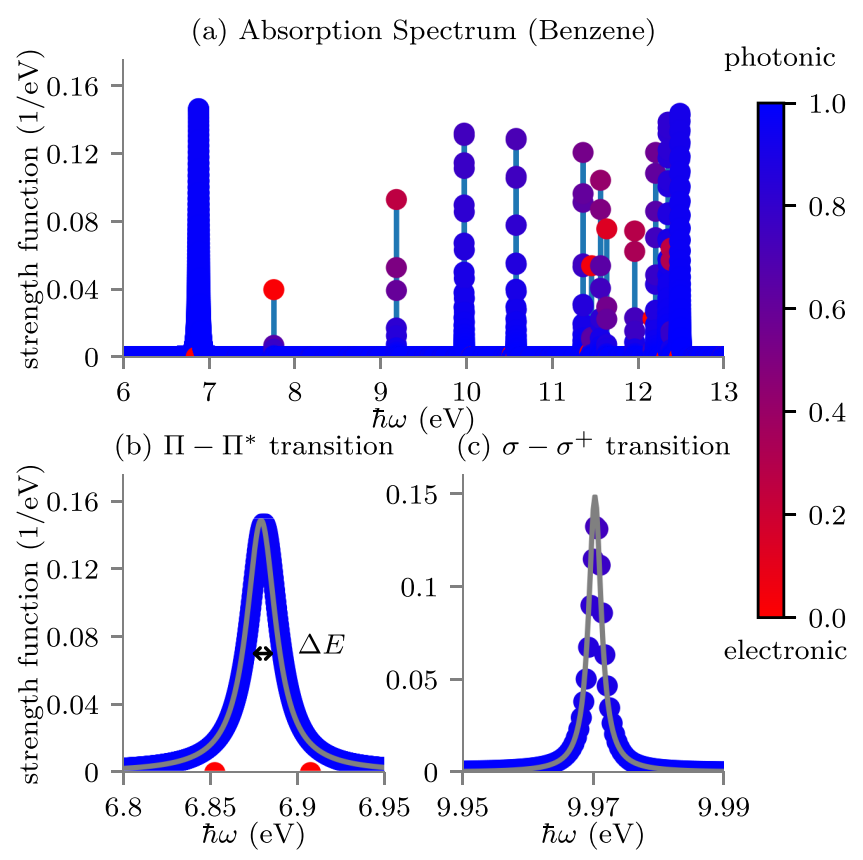

Figure 7. First-principles lifetime calculation of the electronic excitation spectrum of the benzene molecule in an quasi onedimensional cavity: (a) Full spectrum of the benzene molecule, (b) zoom to the $\Pi-\Pi^{*}$ transition, where the black arrow indicates the full width at half-maximum (fwhm) $\Delta E$, (c) zoom to a peak contributing to the $\sigma-\sigma^{+}$transition. The gray spectrum is obtained by WignerWeisskopf theory. ${ }^{31}$ The dotted spectral data points correspond to many coupled electron-photon excitation energies that together comprise the natural line shape of the excitation. Blue color refers to a more photonic nature of the excitations vs red color to a more electronic nature.

and neither subsystem gets appreciably modified due to the other, in contrast to the previous strong light-matter coupling case, the radiative lifetimes of atoms and molecules can be calculated using the perturbative Wigner-Weisskopf theory ${ }^{31}$ in single excitation approximation, as well as under the assumption of the Markov approximation. These approximations are justified in the usual free-space case, where the results of Wigner and Weisskopf reproduce the prior results of Einstein based on the ad-hoc $A$ and $B$ coefficients. However, it does not include the treatment of ensembles of molecules that effectively enhance the matter-photon coupling strength, as shown below. Under the assumption of the Wigner-Weisskopf theory, the radiative decay rate is given by

$$
\Gamma_{3 \mathrm{D}}=\frac{\omega_{0}{ }^{3}|\mathbf{d}|^{2}}{3 \pi \epsilon_{0} \hbar c^{3}}
$$

For a one-dimensional cavity in $\mathrm{x}$-dimension the results change to $^{32}$

$$
\Gamma_{1 \mathrm{D}}=\frac{\omega_{0}|\mathbf{d}|^{2}}{L_{y} L_{z} \epsilon_{0} \hbar c}
$$

For comparison, we show in Figure 7 the peaks in gray that are predicted by the Wigner-Weisskopf theory. Since our sampling is very dense, we find for both peaks shown in the bottom a good agreement with eq 17 .

In fact, if we take the continuum limit for the photon modes, we recover in our framework the lifetimes predicted by the Wigner-Weisskopf theory, including the diverging energy 
shifts, ${ }^{84}$ that is, the Lamb shift. Due to the Lamb shift, our resulting peaks are slightly shifted, due to the divergencies. These divergencies can be handled by renormalization theory. The lifetimes can now be obtained the following way: We measure the full width at half-maximum (fwhm), indicated by the black arrow in (b). In this case, we find $\Delta E_{\text {fwhm }}=0.0204$ $\mathrm{eV}$ and the corresponding lifetime $\tau_{\Pi_{-} \Pi^{*}}$ follows by $\tau_{\Pi-\Pi^{*}}=\hbar$ / $\Delta E_{\mathrm{fwhm}}=32.27 \mathrm{fs}$. Using the Wigner-Weisskopf formula from eq 17 and the dipole moments and energies from the LDA calculation without a photon field, we find a lifetime of 32.21 fs. As a side remark, the same transition using eq 16 has a freespace lifetime of $0.89 \mathrm{~ns}$, roughly in the range of the $2 \mathrm{p}-1 \mathrm{~s}$ lifetime of the hydrogen atom of $1.6 \mathrm{~ns}$.

In Figure $7 c$, we finally show the ab initio peak of the $\sigma-\sigma^{+}$ transition. We find a narrow ab initio peak that is not as well sampled as the $\Pi-\Pi^{*}$. We note in passing that we find an ionization energy of $9.30 \mathrm{eV}$ using $\Delta$-SCF in the benzene molecule with the LDA exchange-correlation functional. In our simulation, coupling to peaks higher than the ionization energy are broadened by continuum (box) states.

3.4. Beyond the Single Molecule Limit and Dissipation in QEDFT. In contrast to the free-space result, where weak coupling as well as the assumption of a dilute gas of molecules are implied, in the case of single-molecule strong coupling $^{8}$ or when nearby molecules or an ensemble of interacting molecules modify the vacuum, the usual perturbative theories break down. Changes in the electronic and the photonic subsystem become self-consistent, and the usual distinction of light and matter becomes less clear. In such situations, the linear-response formulation of QEDFT as well as the Maxwell's perspective of excited-state properties becomes most powerful. Consider, for instance, two benzene molecules weakly coupled to a one-dimensional continuum of photon modes. If the molecules are far apart, we just find the usual Wigner-Weisskopf result. But if we bring the molecules closer (see Figure 8a), we see that the combined resonance shifts and the combined line width becomes broader, implying a shortened lifetime. In Figure $8 \mathrm{~b}$, we consider the case of single-molecule strong coupling, where a few out of the 80000 modes have an enhanced coupling strength. In red, we show the spectrum where the molecule is coupled to the continuum, as is also shown in Figure 7. We then introduce a single strongly coupled mode at the $\Pi-\Pi^{*}$ transition energy, and the resulting spectra is shown in green. We note that, in the figure, the cavity frequencies are plotted in dashed lines. The single mode introduces the expected Rabi splitting into the upper and lower polariton and the peaks of the upper and lower polariton become broadened due to the interaction with the continuum. Interestingly, we find a different line broadening for the lower and the upper polaritonic peak, since only the sum of both has to be conserved. The smaller broadening for these two lower polaritonic states implies that the radiative lifetimes of the lower and upper polaritonic states are longer than the lifetime of the excitation in weakly coupled free-space. In blue, we show the spectra, where we have introduced three strongly coupled modes in addition to the cavity 80000 modes of the continuum. We tune the two additional cavity modes in resonance to the lower and upper polariton peaks of the green plot. We find additional peak splitting, but also a shifting of peak positions, at $7.8 \mathrm{eV}$.

In the last numerical example, we study the strong coupling to the continuum for the case of a single molecule. The results are shown in Figure 9. Here, we effectively enhance the light-

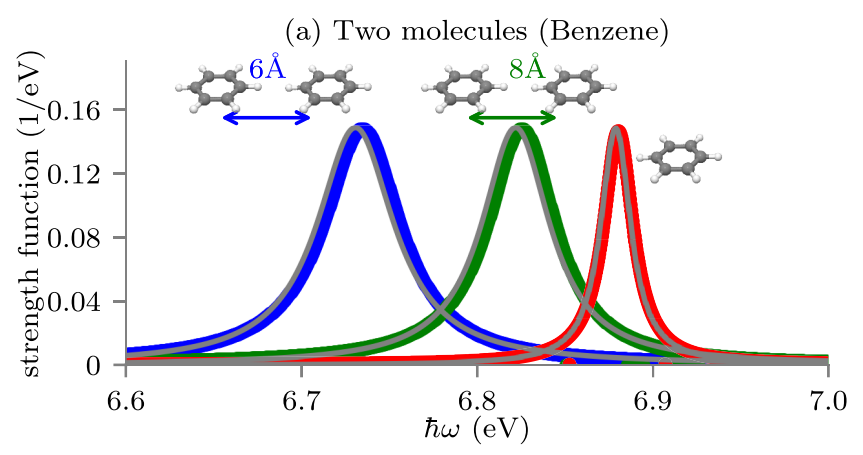

(b) Mulit mode - Rabi Splitting (Benzene)

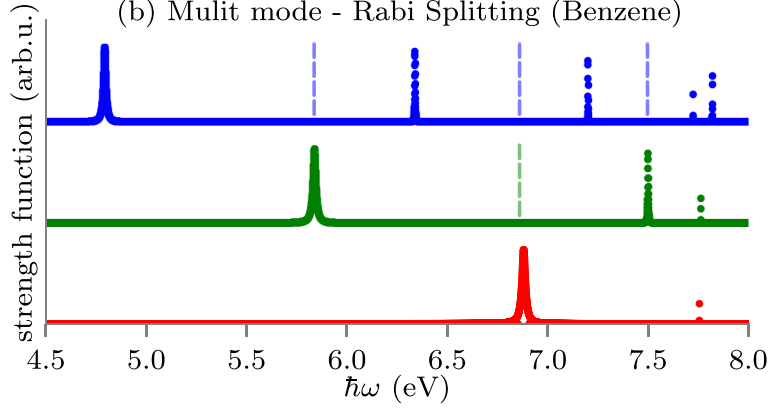

Figure 8. (a) Two molecules of benzene strongly coupled to 80000 cavity modes of an one-dimensional cavity. The further apart the molecules are, the closer the peak gets to the single molecule peak. Also, we notice the doubled peak broadening (shorter lifetime). The gray spectrum is obtained by the Wigner-Weisskopf theory. ${ }^{31}$ (b) We show the Rabi splitting in a situation of a single strongly coupled mode with 80000 cavity modes (green) and three strongly coupled modes with 80000 cavity modes (blue). The red lines correspond to the same setup as in (a). The dashed lines refer to the frequency of the cavity modes. The peaks become broadened due to the interaction with the continuum.

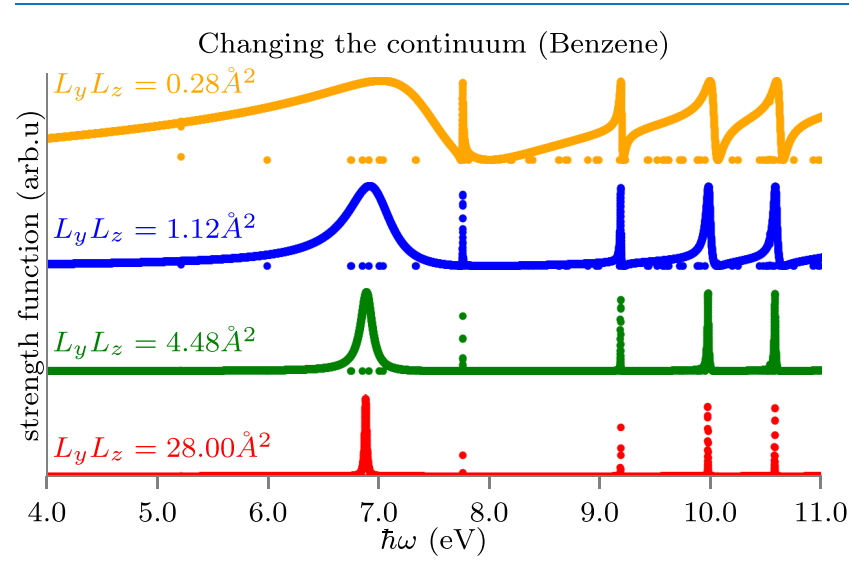

Figure 9. $\mathrm{Ab}$ intio lifetime calculation of the electronic excitation spectrum of the benzene molecule in a one-dimensional cavity along the $x$-direction with different lengths in the $L_{y}$ and $L_{z}$ directions. The red spectra refer to the same setup as in Figure 7. Effectively the electron-photon strength increases with smaller $L_{y}$ and $L_{z}$ lengths leading to a transition from a Lorentzian line shape to a Fano line shape.

matter coupling strength by reducing the volume of the cavity along the $y$ - and $z$-direction. For comparison, we show in red the setup that is also shown in Figure 7, where the excitations have a Lorentzian line shape consistent with the WignerWeisskopf theory, as discussed in the previous section. By gradually reducing the dimensions along the $y$ - and $z$-direction, we find drastic changes in the line shape of the excitations. 
These changes lead to the transition of the line shape from a Lorentzian to a Fano line shape, as becomes clearly visible for $L_{x} L_{z}=0.28 \AA$.

As a summary, we have presented in this section, that lineshapes, as well as lifetimes can be inferred directly from first principle calculations. In the case of a Lorentzian line shape, we find that the width of the calculated peaks (no need to introduce any artificial broadening as commonly done) correspond to the lifetimes. These calculations demonstrate that the $a b$ initio theory is able to capture the true nature of excitations, that is, resonances with finite intrinsic lifetimes, without the need of an artificial bath or postprocessing. Furthermore, we find that the excitations measured in absorption/emission experiments are mainly photonic in nature, and it is only the peak position that is dominated by the matter constituents. This is, of course, very physical, since what we see is the absorption/emission of a photon, not of the matter constituents. Further, since we describe the photon vacuum on the same theoretical footing as the matter subsystem, we have full control over the photon field, making it straightforward to simulate very intricate changes, for example, changing the character of a specific mode out of basically arbitrarily many, and investigating its influence on excited-state properties such as the radiative lifetime. This allows predictive first-principle calculations for intricate experimental situations similar to the ones encountered in refs 33 and 34.

\section{SUMMARY AND OUTLOOK}

In this work we have introduced a linear-response theory for nonrelativistic quantum-electrodynamics in the long wavelength limit, which can be straightforwardly extended to the full minimal coupling case. Compared to the conventional matter-only response approaches, we have highlighted how in the coupled matter-photon case the usual response functions change, how photon-photon and matter-photon response functions are introduced, how these response functions provide a photonic perspective on excited state properties, how the results lead to self-consistent Maxwell's equation in matter, and how we can efficiently calculate all these response functions in the framework of QEDFT. By investigating a simple model system, we have shown how the spectrum of the matter subsystem is changed upon coupling to the photon field. Further, we have demonstrated the range of validity of a simple yet reliable approximation to the, in general, unknown mean-field exchange-correlation kernels. Using this approximation, we have presented the first ab initio calculations of the spectrum of real systems (benzene molecules) coupled to the modes of the quantized electromagnetic field. In one example we have calculated the change upon strong coupling to a single mode of a high- $Q$ cavity, which leads to a large Rabi splitting. In the second example we have calculated from first-principles the natural line widths of benzene coupled to a specific sampling of the vacuum field. In the last examples, we demonstrated the abilities to calculate many-molecule systems, as well as dissipative strong-coupling situations, as well as strong coupling to the continuum, where we find a transition from Lorentzian line shape to Fano line shape, where the usual (perturbative) approaches to light-matter coupling fail. These results demonstrate the versatility and possibilities of QEDFT, where light and matter are treated on equal quantized footing. In the context of strong light-matter coupling, for example, in polaritonic chemistry, the presented linear-response formula- tion allows now to determine polaritonically modified spectra from first principles. Together with $a b$ initio ground-state calculations, ${ }^{39}$ QEDFT now provides a workable first-principle description to analyze and predict photon-dressed chemistry and material sciences. In particular, our approach provides a unique practical computational scheme to compute photondressed excited-state potential-energy surfaces and nonadiabatic coupling elements ${ }^{49}$ that are required for ab initio calculations in the emerging field of polaritonic chemistry. Further, in the context of standard ab initio theory, the linearresponse formulation of QEDFT now allows the calculation of intrinsic lifetimes and provides access to quantum-optical observables. Specifically, due to the nonperturbative nature of the approach, quantum-optical problems where the selfconsistent feedback between light and matter has to be taken into account, for example, that many molecules change the photon vacuum and hence the Markov approximation breaks down, becoming feasible. For optical physics, the presented linear-response framework presents an interesting opportunity to study the self-consistency of the Maxwell's equations in matter from first principles. Finally, we want to highlight that although the QEDFT linear-response framework includes the coupling of light and matter, its similarity to the usual matteronly linear-response formulation in terms of a pseudoeigenvalue problem makes it very easy to include in already existing first-principle codes. This, together with the above-discussed possibilities in different fields of physics, shows that there are many interesting cases that can be studied with the presented method.

\section{SELF-CONSISTENCY OF THE MAXWELL'S EQUATION}

In this section we give more details on the modifications of the Maxwell's equations. The semiclassical description of the light-matter interaction is limited as a result of the transverse field being treated as an external perturbation. This approximation breaks the feedback loop between light and matter that leads to self-consistency in the Maxwell's equation. Let us start from the classical description and assume that we are interested in the induced fields due to an external perturbation. If everything is perfectly classical there is no difference whether we perturb by an external transversal field $\mathbf{a}_{\perp}$ or an external classical current $\mathbf{j}_{\perp}$ due to the inhomogeneous Maxwell's equation in vacuum

$$
\left(\frac{1}{c^{2}} \frac{\partial^{2}}{\partial t^{2}}-\nabla^{2}\right) \mathbf{a}_{\perp}(\mathbf{r}, t)=\mu_{0} c \mathbf{j}_{\perp}(\mathbf{r}, t)
$$

Now, if we have some theory to relate these external perturbations to the induced current $\mathrm{J}_{\perp}\left[\mathbf{a}_{\perp}\right]$, the induced field reads

$$
\left(\frac{1}{c^{2}} \frac{\partial^{2}}{\partial t^{2}}-\nabla^{2}\right) \mathbf{A}_{\perp}(\mathbf{r}, t)=\mu_{0} c \mathbf{J}_{\perp}\left(\left[\mathbf{a}_{\perp}\right], \mathbf{r}, t\right)
$$

from which we can calculate the induced physical fields, for example, the transversal electric field in Coulomb gauge is $\mathbf{E}_{\perp}(\mathbf{r}, t)=-1 / c \partial_{t} \mathbf{A}_{\perp}(\mathbf{r}, t)$ (some textbooks ${ }^{44}$ define the connection of the electric field to the vector potential without the prefactor $1 / c$; we use the current notation to be consistent with relativistic literature and ref 37 ). We can again combine these two results and look at the total field $\mathbf{A}_{\perp}^{\text {tot }}=\mathbf{a}_{\perp}+\mathbf{A}_{\perp}$, which obeys 


$$
\left(\frac{1}{c^{2}} \frac{\partial^{2}}{\partial t^{2}}-\nabla^{2}\right) \mathbf{A}_{\perp}^{\mathrm{tot}}(\mathbf{r}, t)=\mu_{0} c\left(\mathbf{j}_{\perp}(\mathbf{r}, t)+\mathbf{J}_{\perp}\left(\left[\mathbf{j}_{\perp}\right], \mathbf{r}, t\right)\right)
$$

Using the Maxwell relations once more, we can equivalently find, for example, the induced electric field

$$
\left(\frac{1}{c^{2}} \frac{\partial^{2}}{\partial t^{2}}-\nabla^{2}\right) \mathbf{E}_{\perp}(\mathbf{r}, t)=-\mu_{0} \frac{\partial}{\partial t} \mathbf{J}_{\perp}\left(\left[\mathbf{a}_{\perp}\right], \mathbf{r}, t\right)
$$

We can now make a connection to the Maxwell's equation in matter, where $\mathbf{j}_{\perp}$ is called the free current and $\mathbf{J}_{\perp}$ is the bound current. Assuming that we can express the induced transversal current locally around the center of charge as $\mathbf{J}_{\perp}(\mathbf{r}, t) \approx \frac{\partial}{\partial t} \mathbf{P}_{\perp}(\mathbf{r}, t)$, where we use the polarization

$$
\mathbf{P}_{\perp}(\mathbf{r}, t)=\epsilon_{0} e \sum_{\alpha=1}^{M} \lambda_{\alpha}(\mathbf{r}) \int \mathrm{d} \mathbf{r}^{\prime} \lambda_{\alpha}\left(\mathbf{r}^{\prime}\right) \cdot \mathbf{r}^{\prime} n\left(\left[\mathbf{a}_{\perp}\right], \mathbf{r}^{\prime}, t\right)
$$

and expand the electric field in the modes $\lambda_{\alpha}(\mathbf{r})$ as

$$
\mathbf{E}_{\perp}(\mathbf{r}, t)=\sum_{\alpha=1}^{M} \lambda_{\alpha}(\mathbf{r}) E_{\alpha}(t)
$$

we can rewrite the above equation at the center of charge, that is, $\lambda_{\alpha}(\mathbf{r}) \rightarrow \lambda_{\alpha}$, as

$$
\left(\frac{\partial^{2}}{\partial t^{2}}+\omega_{\alpha}^{2}\right) E_{\alpha}(t)=-\frac{\partial^{2}}{\partial t^{2}} \lambda_{\alpha} \cdot \mathbf{R}\left(\left[\mathbf{a}_{\perp}\right], t\right)
$$

Using this kind of approach, we can connect $\delta n(\mathbf{r}, t)$ of eq 3 to the induced electric field $\delta \mathbf{E}_{\perp}(\mathbf{r}, t)$, where we employ a spatially homogeneous vector potential $\mathbf{a}_{\perp}(t)$ that gives rise to the external electric field $\mathbf{E}_{\perp}^{\text {ext }}(t)=-\frac{1}{c} \frac{\partial}{\partial t} \mathbf{a}_{\perp}(t)$. In a final step, to avoid solving the above mode-resolved Maxwell's equations, one often even ignores the spatial dependence of the induced field and merely uses $E_{\alpha}(t)=-\lambda_{\alpha} \cdot \mathbf{R}\left(\left[\mathbf{a}_{\perp}\right], t\right)$. If we now determine in the linear response $\mathbf{R}\left(\left[\mathbf{a}_{\perp}\right], t\right)$, we immediately see that when $\chi_{n}^{n}$ is changed due to strong light-matter coupling, also the induced field is changed. Furthermore, the reformulation of the linear-response kernel in eq 10 shows that we get a feedback from the induced photon field onto the matter. Such intrinsic back-reaction (screening) effects are very important for large systems, as is well-known from solid-state physics, where the bare (vacuum) electric field, as determined by eq 23 , does not agree with the measured spectrum. One needs to include the self-consistent polarization of the system that counteracts the external perturbing field. This can be done approximately in linear response by solving self-consistently a Maxwell's equation with the matter response as input. ${ }^{66-69}$ In the theory of classical electrodynamics, a convenient way to do so is to switch to the Maxwell's equations in matter. In the above considerations, this means we introduce the displacement field $\mathbf{D}_{\perp}=\epsilon_{0} \mathbf{E}_{\perp}+\mathbf{P}_{\perp}$, where now all the knowledge about how the system reacts to an external perturbation is encoded again in $\mathbf{P}_{\perp}$, such that we find

$$
\left(\frac{1}{c^{2}} \frac{\partial^{2}}{\partial t^{2}}-\nabla^{2}\right) \mathbf{D}_{\perp}(\mathbf{r}, t)=-\nabla^{2} \mathbf{P}_{\perp}\left(\left[\mathbf{a}_{\perp}\right], \mathbf{r}, t\right)
$$

After expanding $\mathbf{D}_{\perp}(\mathbf{r}, t)=\epsilon_{0} \sum_{\alpha} \omega_{\alpha} \lambda_{\alpha}(\mathbf{r}) q_{\alpha}(t)$ and then performing the long wavelength limit, we arrive at

$$
\left(\frac{\partial^{2}}{\partial t^{2}}+\omega_{\alpha}^{2}\right) q_{\alpha}(t)=\omega_{\alpha} \lambda_{\alpha} \cdot \mathbf{R}\left(\left[\mathbf{a}_{\perp}\right], t\right)
$$

which is the classical analogue of eq 8 . In the usual decoupled light-matter description without self-consistency, we then simply determine $\mathbf{R}\left(\left[\mathbf{a}_{\perp}\right], t\right)$ from the electric permittivity and ignore any feedback that describes how the matter system affects (screens) the field. Approximate self-consistency is found once the induced field $\mathbf{E}_{\perp}$ is taken into account to screen the perturbing field $\mathbf{E}_{\perp}^{\text {ext }}$. But, in our case, we want to go beyond this simple approximate self-consistency, which will break down once the coupling between light and matter is strong. Note that, in the macroscopic Maxwell's equation, the electric field becomes $E_{\alpha}(t)=\omega_{\alpha} q_{\alpha}(t)-\lambda_{\alpha} \cdot \mathbf{R}\left(\left[\mathbf{a}_{\perp}\right], t\right)$, and we see that if we ignore the spatial dependence in determining $E_{\perp}$, we basically assume $\mathbf{D}_{\perp}=\mathbf{E}_{\perp}$.

In our description, we keep the photon field as a dynamical variable of the system, such that the Maxwell field couples to the electronic system, leading to a fully self-consistent description of the light-matter response. Besides the changes in $\chi_{n}^{n}$, which when used as an input into eqs 21 or 25 , captures the self-consistent response of the light-matter system, we can now also directly access the induced electric field by considering the response of the displacement field due to $\chi_{n}^{q}$ and the use of

$$
\hat{\mathbf{E}}_{\perp}=\sum_{\alpha=1}^{M} \lambda_{\alpha} \omega_{\alpha}\left(\hat{q}_{\alpha}-\frac{\lambda_{\alpha}}{\omega_{\alpha}} \cdot \mathbf{R}\right)
$$

As discussed in section 1.4, this leads to a complete change of perspective, since it highlights that the excited states of the coupled light-matter system can be viewed as changes in the quantized Maxwell field in accordance to the usual experimental situation. On the other hand, we can now also investigate what the quantum description of the coupled lightmatter system does to the Maxwell's equations. We therefore consider the case where the free (time-derivative of the) current $\delta j_{\alpha}(t)$ is nonzero while the external classical field is zero, that is, $\delta v(\mathbf{r}, t)=0$. In this case, we find

$$
\begin{aligned}
& \left(\frac{\partial^{2}}{\partial t^{2}}+\omega_{\alpha}^{2}\right) \delta q_{\alpha}(t)=-\frac{\delta j_{\alpha}(t)}{\omega_{\alpha}}+\omega_{\alpha} \boldsymbol{\lambda}_{\alpha} \cdot \\
& \int e \mathbf{r} \chi_{n, \mathrm{~s}}^{n} f_{\mathrm{Mxc}}^{n} \chi_{q_{\alpha}}^{n} \delta j_{\alpha}+\omega_{\alpha} \boldsymbol{\lambda}_{\alpha} \cdot \bigvee e \mathbf{r} \chi_{n, \mathrm{~s}}^{n} f_{\mathrm{Mxc}}^{q_{\alpha^{\prime}}} \delta q_{\alpha^{\prime}}
\end{aligned}
$$

If we contrast this to the classical Maxwell's equation in matter

$$
\left(\frac{\partial^{2}}{\partial t^{2}}+\omega_{\alpha}^{2}\right) \delta q_{\alpha}(t)=-\frac{\delta j_{\alpha}(t)}{\omega_{\alpha}}+\omega_{\alpha} \lambda_{\alpha} \cdot \delta \mathbf{R}\left(\left[\mathbf{j}_{\perp}\right], t\right)
$$

where $\mathbf{R}\left(\left[\mathbf{j}_{\perp}\right], t\right)$ would be determined from the response of the matter system due to the corresponding external field $\mathbf{a}_{\perp}$, we see that, besides the self-consistent response of the matter system (second term on the right-hand side), also a genuine (matter-mediated) photon-photon interaction term (third term on the right-hand side) appears. Making again the meanfield explicitly lead to 


$$
\begin{aligned}
& \left(\frac{\partial^{2}}{\partial t^{2}}+\omega_{\alpha}^{2}\right) \delta q_{\alpha}(t)=-\frac{\delta j_{\alpha}(t)}{\omega_{\alpha}}+\omega_{\alpha} \boldsymbol{\lambda}_{\alpha} \cdot \\
& \int e \mathbf{r} \chi_{n, \mathrm{~s}}^{n}\left[\frac{e^{2}}{4 \pi \epsilon_{0}\left|\mathbf{r}^{\prime}-\mathbf{r}^{\prime \prime}\right|}+\sum_{\alpha^{\prime}}\left(\boldsymbol{\lambda}_{\alpha^{\prime}} \cdot e \mathbf{r}^{\prime \prime}\right) \boldsymbol{\lambda}_{\alpha^{\prime}} \cdot e \mathbf{r}^{\prime}\right] \chi_{q_{\alpha}}^{n} \delta j_{\alpha} \\
& -\omega_{\alpha} \boldsymbol{\lambda}_{\alpha} \cdot \bigvee e \mathbf{r} \chi_{n, \mathrm{~s}}^{n}\left(\omega_{\alpha^{\prime}} \boldsymbol{\lambda}_{\alpha^{\prime}} \cdot e \mathbf{r}^{\prime}\right) \delta q_{\alpha^{\prime}}+\omega_{\alpha} \boldsymbol{\lambda}_{\alpha} \cdot \\
& \int e \mathbf{r} \chi_{n, \mathrm{~s}}^{n} f_{\mathrm{xc}}^{n} \chi_{q_{\alpha}}^{n} \delta j_{\alpha}+\omega_{\alpha} \boldsymbol{\lambda}_{\alpha} \cdot \bigvee e \mathbf{r} \chi_{n, \mathrm{~s}}^{n} f_{\mathrm{xc}}^{q_{\alpha^{\prime}}} \delta q_{\alpha^{\prime}}
\end{aligned}
$$

If we ignore the $\mathrm{xc}$ contributions to the matter-photon and photon-photon response, we get the pRPA approximation to the Maxwell's equation in matter. In this pRPA form, we clearly see how the Maxwell's equation becomes nonlinear because of the feedback between light and matter. Such nonlinearities of the Maxwell's equations are investigated in great detail in high-energy physics in the context of strong-field QED. ${ }^{85}$ In that case, the strong fields lead to particle creation and, thus, a matter-mediated photon-photon interaction. In our case, we do not need these high energies, because we consider the photon-photon interaction due to condensed matter in the form of atoms, molecules, or solids and use, for example, a cavity to enhance the coupling. That the changes in the Maxwell's equations are not purely theoretical concepts, but lead to observable effects can be seen in many physical situations. As mentioned before, the most well-known effect are polarization effects in solid-state systems, ${ }^{69}$ but more strikingly are effects due to the quantum-matter-mediated photon-photon interactions, see, for example, ref 86 . In this context, the presented ab initio method allows to theoretically investigate the photon-photon interactions and possibly predict systems with very strong photon-photon correlations. In such cases, the strong photon correlations could be used to give complementary insights into molecular systems or to imprint the photonic correlations on the matter subsystem. Besides these differences, we highlight that the quantized Maxwell's equation in matter, if we allow for both, a free external current and a free external field, can indeed discriminate between these two sorts of perturbations. In a purely classical theory, due to eq 18 , there can be no difference. This provides a playground to investigate the difference between classical and quantum physics.

\section{PHOTONIC OBSERVABLES AND RADIATIVE LIFETIMES}

In the presented framework, besides the above highlighted changes in, for example, the Maxwell's equations, a wealth of interesting observables become accessible. For instance, one can monitor the response of the matter system due to a perturbation of the photonic subsystem by an external current. This allows to investigate directly the cross-correlation between the matter and the photon subsystem induced by $\chi_{q_{\alpha}}^{n}$. Also note that this cross-correlation observable allows to distinguish between the response due to a purely classical field $\delta v(\mathbf{r}, t)$ or due to a quantized field, since $\delta j_{\alpha}(t)$ generates photons (which is equivalent to just use a slightly different initial state with an incoming photon pulse) that then perturb the correlated matter-photon system. This makes the presented framework applicable to also determine observables due to novel spectroscopies that use quantum light. ${ }^{87}$ This area of spectroscopy is so far not accessible with common first- principle methods. One further important observable that can be captured in this approach is the intrinsic lifetimes of excited states, which are not accessible in standard matter-only quantum mechanics. Let us briefly explain what we mean by this. In standard quantum mechanics, we find besides the ground state also other eigenstates, that is, excited states. Hereby, an eigenstate is a square-integrable eigenfunction of a self-adjoint, usually unbounded Hamiltonian. If we excite a matter system from its ground state into such an excited state, it will remain in this state as long as we do not perturb it. In quantum mechanics we then also have generalized eigenstates, so-called scattering states, which are not square-integrable and that constitute the continuous spectrum of such a Hamiltonian. ${ }^{88}$ The simplest example is the free electronic Hamiltonian $\hat{T}=\sum_{i=1}^{N}-\frac{\hbar^{2}}{2 m_{e}} \nabla_{i}^{2}$ which in infinite space has a purely continuous spectrum consisting of non-normalizable plane-waves. ${ }^{89}$ The physical interpretation of such scattering states - as already the name indicates - is that particles propagate to infinity and do not stay bound anywhere. Thus, exciting a matter system from its ground state into such a generalized eigenstate corresponds to the physical process of ionization. Ionization, however, is something completely different than the process of spontaneous emission. That is, if we put an atom or molecule into an "excited state", even without a further perturbation it will relax to the ground state by emitting radiation. The time the system stays in this "excited state" before emitting a photon is called the lifetime. The process of spontaneousemission clearly cannot be captured by standard quantum mechanics where matter and light are decoupled. Nonrelativistic QED, however, does capture this process ${ }^{45}$ by coupling the matter system to the quantized electromagnetic field which consists of infinitely many harmonic oscillators. In this way the excited states of the bare matter system turn into resonances and the ground state (usually) remains the only eigenstate of the combined matterphoton system. While formally these resonances are indeed scattering states of the combined matter-photon system, it is only the photonic part that shows a scattering behavior, i.e., a photon leaves the vicinity of the matter subsystem. ${ }^{32,83}$ The matter subsystem just relaxes to the only stable state, its ground state. ${ }^{45}$ In linear response such relaxation processes express themselves as finite line widths of excitations, where the line width can be associated with the lifetimes of the different resonances. In our slightly simplified treatment based on eq 1 , we only consider a finite number of photon modes, and hence, we do not have genuine resonances. However, by including enough modes, we sample the influence of the vacuum, and instead of one sharp transition peak (which numerically is usually artificially broadened), we get many that approximate the resonance. In this way, the linear-response theory for nonrelativistic QED in the long wavelength limit can determine lifetimes of real systems. We show an example for such an ab initio lifetime calculation in section 3.2. This provides an interesting field of research that the presented framework makes it accessible for ab initio theory. While it is conceptually very interesting to revisit well-known results for intrinsic radiative lifetimes of gas phase molecules, since we can now study the nature of resonances in detail (see, e.g., the discussion on the photonic nature of resonances in section 3.2), we now have access to even more exciting experimental situations. By changing the environment, for example, putting the molecule inside a cavity and thus enhance certain modes 
while suppressing others, one can change and control the radiative lifetimes of single molecules ${ }^{33,34}$ (see also section 3.4). We can thus theoretically study and predict realistic experimental situations where nontrivial changes in the photonic vacuum, for example, due to nearby surfaces or other physical entities, directly influence intrinsic lifetimes and properties of resonances.

\section{LINEAR-RESPONSE THEORY AS A PSEUDOEIGENVALUE PROBLEM}

In this section, we reformulate the linear-response theory of coupled electron-photon systems as a pseudoeigenvalue problem. The entire linear-response in nonrelativistic QED for the density and photon coordinate can be written in the matrix form as

$$
\left(\begin{array}{c}
\delta n \\
\delta q_{1} \\
\delta q_{2} \\
\vdots \\
\delta q_{M}
\end{array}\right)=\left(\begin{array}{ccccc}
\chi_{n}^{n} & \chi_{q_{1}}^{n} & \chi_{q_{2}}^{n} & \ldots & \chi_{q_{M}}^{n} \\
\chi_{n}^{q_{1}} & \chi_{q_{1}}^{q_{1}} & \chi_{q_{2}}^{q_{1}} & \ldots & \chi_{q_{M}}^{q_{1}} \\
\chi_{n}^{q_{2}} & \chi_{q_{1}}^{q_{2}} & \chi_{q_{2}}^{q_{2}} & \ldots & \chi_{q_{M}}^{q_{2}} \\
\vdots & \vdots & \vdots & \ddots & \vdots \\
\chi_{n}^{q_{M}} & \chi_{q_{1}}^{q_{M}} & \chi_{q_{2}}^{q_{M}} & \ldots & \chi_{q_{M}}^{q_{M}}
\end{array}\right)\left(\begin{array}{c}
\delta v \\
\delta j_{1} \\
\delta j_{2} \\
\vdots \\
\delta j_{M}
\end{array}\right)
$$

where we imply integration over time and space when appropriate. In this form we clearly see that the density response of the coupled matter-photon system depends on whether we use a classical field $\delta v(\mathbf{r}, t)$, photons, which are created by $\delta j_{\alpha}(t)$, or combinations thereof, for the perturbation. The explicit coupling between the subsystems (i.e., matter and photons) demonstrates changes in the subsystems as a result of the back-reaction between matter and photons. The cross-talk between the respective coupled subsystems shows up in the cross-correlation response functions, which leads to changes in the respective observables $\left(n(\mathbf{r}, t), q_{\alpha}(t)\right)$. This becomes evident by considering an external perturbation of the coupled system with the external potential $\delta v(\mathbf{r} t)$ reduces to the coupled set of responses

$$
\left\{\begin{array}{l}
\delta n(\mathbf{r} t)=\iint \mathrm{d} t^{\prime} \mathrm{d} \mathbf{r}^{\prime} \chi_{n}^{n}\left(\mathbf{r} t, \mathbf{r}^{\prime} t^{\prime}\right) \delta v\left(\mathbf{r}^{\prime} t^{\prime}\right) \\
\delta q_{\alpha}(t)=\iint \mathrm{d} t^{\prime} \mathrm{d} \mathbf{r}^{\prime} \chi_{n}^{q_{\alpha}}\left(t, \mathbf{r}^{\prime} t^{\prime}\right) \delta v\left(\mathbf{r}^{\prime} t^{\prime}\right)
\end{array}\right.
$$

Here, the cross-correlation response function $\chi_{n}^{q_{\alpha}}\left(t, \mathbf{r}^{\prime} t^{\prime}\right)$ accounts for the action of the matter subsystem on the photon field which gives rise to a response of the photon field as a result of perturbing the matter. In the semiclassical approach in which TDDFT is based on, the cross-correlation response function do not show up but rather just a simplified form (since there the wave function describes only the matter subsystem) of the $\chi_{n}^{n}\left(\mathbf{r} t, \mathbf{r}^{\prime} t^{\prime}\right)$. Similarly, a perturbation of the coupled system with the external charge current $\delta j_{\alpha}(t)$ results in

$$
\left\{\begin{array}{l}
\delta n(\mathbf{r} t)=\sum_{\alpha=1}^{M} \int \mathrm{d} t^{\prime} \chi_{q_{\alpha}}^{n}\left(\mathbf{r} t, t^{\prime}\right) \delta j_{\alpha}\left(t^{\prime}\right), \\
\delta q_{\alpha}(t)=\sum_{\alpha^{\prime}=1}^{M} \int \mathrm{d} t^{\prime} \chi_{q_{\alpha^{\prime}}}^{q_{\alpha}}\left(t, t^{\prime}\right) \delta j_{\alpha^{\prime}}\left(t^{\prime}\right)
\end{array}\right.
$$

The cross-correlation response function $\chi_{q_{\alpha}}^{n}\left(\mathbf{r} t, t^{\prime}\right)$ accounts for the action of the photon field on the matter, thus, specifying the response of the density by perturbing the photon field, and $\chi_{q_{\alpha}^{\prime}}^{q_{\alpha}}\left(t, t^{\prime}\right)$ describes how the photon interacts via matter, thus, specifying changes in response of the photon field.

Next, we need to find an efficient way to solve these linearresponse equations in terms of the Maxwell KS system. First, performing a Fourier transformation from time $t$ and $t^{\prime}$ to frequency space $\omega$ and using the Hxc and pxc kernels, we write the response functions of eqs 10-13 in the following compact notation

$$
\begin{aligned}
& \chi_{n}^{n}=\chi_{n, \mathrm{~s}}^{n}+\chi_{n, \mathrm{~s}}^{n}\left[\left(f_{\mathrm{pxc}}^{n}+f_{\mathrm{Hxc}}^{n}\right) \chi_{n}^{n}+\sum_{\alpha} f_{\mathrm{pxc}}^{q_{\alpha}} \chi_{n}^{q_{\alpha}}\right] \\
& \chi_{n}^{q_{\alpha}}=\sum_{\beta} \chi_{q_{\beta, \mathrm{s}}}^{q_{\alpha}} g_{\mathrm{M}}^{n_{\beta}} \chi_{n}^{n} \\
& \chi_{q_{\alpha}}^{n}=\chi_{n, \mathrm{~s}}^{n}\left[\sum_{\alpha^{\prime}} f_{\mathrm{pxc}}^{q_{\alpha^{\prime}}} \chi_{q_{\alpha}}^{q_{\alpha^{\prime}}}+\left(f_{\mathrm{pxc}}^{n}+f_{\mathrm{Hxc}}^{n}\right) \chi_{q_{\alpha}}^{n}\right] \\
& \chi_{q_{\alpha^{\prime}}}^{q_{\alpha}}=\chi_{q_{\alpha^{\prime}, \mathrm{s}}}^{q_{\alpha}}+\sum_{\beta} \chi_{q_{\beta^{\prime}}, s_{\mathrm{M}}}^{q_{\alpha}} \chi_{q_{\alpha^{\prime}}}^{n_{\beta}} \chi^{n}
\end{aligned}
$$

Those equations are coupled with respect to the external perturbations as seen in eqs 29 and 30. The perturbation with respect to the external potential $\delta v(\mathbf{r} t)$ results in a coupled set of response functions $\left\{\chi_{n}^{n}\left(\mathbf{r} t, \mathbf{r}^{\prime} t^{\prime}\right), \chi_{n}^{q_{\alpha}}\left(t, \mathbf{r}^{\prime} t^{\prime}\right)\right\}$ and for the external current $\delta j_{\alpha}(t)$ gives the coupled set $\left\{\chi_{q_{\alpha}}^{n}\left(\mathbf{r} t, t^{\prime}\right), \chi_{q_{\alpha}{ }^{\prime}}^{q_{\alpha}}(t\right.$, $\left.\left.t^{\prime}\right)\right\}$. These pairs of coupled response functions have to be solved in a self-consistent way to obtain the exact interacting response functions. The response functions of eqs 29 and 30 can be expressed in frequency space through a Fourier transform that yields

$$
\begin{aligned}
& \delta n_{v}(\mathbf{r}, \omega)=\int \mathrm{d} \mathbf{r}^{\prime} \chi_{n}^{n}\left(\mathbf{r}, \mathbf{r}^{\prime}, \omega\right) \delta v\left(\mathbf{r}^{\prime}, \omega\right) \\
& \delta q_{\alpha, v}(\omega)=\int \mathrm{d} \mathbf{r}^{\prime} \chi_{n}^{q_{\alpha}}\left(\omega, \mathbf{r}^{\prime}\right) \delta v\left(\mathbf{r}^{\prime}, \omega\right) \\
& \delta n_{j}(\mathbf{r}, \omega)=\sum_{\alpha} \chi_{q_{\alpha}^{n}}^{n}(\mathbf{r}, \omega) \delta j_{\alpha}(\omega) \\
& \delta q_{\alpha, j}(\omega)=\sum_{\alpha^{\prime}} \chi_{q_{\alpha^{\prime}}}^{q_{\alpha}}(\omega) \delta j_{\alpha^{\prime}}(\omega)
\end{aligned}
$$

A perturbation with the external potential $\delta v(\mathbf{r}, \omega)$ induces the responses $\delta n_{v}(\mathbf{r}, \omega)$ and $\delta q_{\alpha, v}(\omega)$. Making a substitution of eqs 31 and 32 into the density and displacement field response due to an external potential $\delta v(\mathbf{r}, \omega)$ yields, after some algebra, the following eigenvalue problem (for a detailed derivation, we refer the reader to the SI, section S4)

$$
\left(\begin{array}{cccc}
L\left(\Omega_{q}\right) & K\left(\Omega_{q}\right) & M\left(\Omega_{q}\right) & M\left(\Omega_{q}\right) \\
K^{*}\left(\Omega_{q}\right) & L\left(\Omega_{q}\right) & M^{*}\left(\Omega_{q}\right) & M^{*}\left(\Omega_{q}\right) \\
N & N^{*} & \omega_{\alpha} & 0 \\
N & N^{*} & 0 & \omega_{\alpha}
\end{array}\right)\left(\begin{array}{l}
\mathbf{X}_{1}\left(\Omega_{q}\right) \\
\mathbf{Y}_{1}\left(\Omega_{q}\right) \\
\mathbf{A}_{1}\left(\Omega_{q}\right) \\
\mathbf{B}_{1}\left(\Omega_{q}\right)
\end{array}\right)=\Omega_{q}\left(\begin{array}{cccc}
1 & 0 & 0 & 0 \\
0 & -1 & 0 & 0 \\
0 & 0 & 1 & 0 \\
0 & 0 & 0 & -1
\end{array}\right)\left(\begin{array}{l}
\mathbf{X}_{1}\left(\Omega_{q}\right) \\
\mathbf{Y}_{1}\left(\Omega_{q}\right) \\
\mathbf{A}_{1}\left(\Omega_{q}\right) \\
\mathbf{B}_{1}\left(\Omega_{q}\right)
\end{array}\right)
$$

In this equation, $\mathbf{X}_{1}$ and $\mathbf{Y}_{1}$ are the contributions to the full solution in the matter part of the equation, while $\mathbf{A}_{1}$ and $\mathbf{B}_{1}$ are the contributions to the solution in the photon part of the equation. Further, $\Omega_{q}$ refers to the many-body electron-photon 
excitation energies. In comparison to the standard linearresponse formulation of TDDFT, new $2 \times 2$ blocks arises, the $M$-block accounts for the explicit electron-photon interaction, the $N$-block accounts for the dipole coupling of the electronic system to the photon field and the $\omega_{\alpha}$-block are the frequencies of the photon field. The quantity $L_{a i, j b}\left(\Omega_{q}\right)=$ $\delta_{a b} \delta_{i j}\left(\epsilon_{a}-\epsilon_{i}\right)+K_{a i, j b}\left(\Omega_{q}\right)$ contains the difference of two Kohn-Sham energies $\epsilon_{a}$ and $\epsilon_{i}$, where $i$ refers to occupied orbitals and the index $a$ to unoccupied orbitals. The couplingmatrix $K$ is given by

$$
K_{a i, \mathrm{~b}}\left(\Omega_{q}\right)=\iint \operatorname{d} \mathbf{r d} \mathbf{y} \varphi_{i}(\mathbf{r}) \varphi_{a}^{*}(\mathbf{r}) f_{\mathrm{Mxc}}^{n}\left(\mathbf{r}, \mathbf{y}, \Omega_{q}\right) \varphi_{b}(\mathbf{y}) \varphi_{j}^{*}(\mathbf{y})
$$

The quantity $K_{a, i j b}\left(\Omega_{q}\right)$ differs from the electron-only case since $f_{\mathrm{Mxc}}^{n}=f_{\mathrm{Hxc}}^{n}+f_{\mathrm{pxc}}^{n}$. Treating the photon field only externally reduces this matrix to the standard coupling matrix in TDDFT linear response with $f_{\mathrm{Mxc}}^{n}=f_{\mathrm{Hxc}}^{n}$. The two new coupling functions appearing, $M$ and $N$ that couple the matter block are given explicitly as

$$
\begin{aligned}
& M_{\alpha, a i}\left(\Omega_{q}\right)=\int \operatorname{d} \mathbf{r} \varphi_{i}(\mathbf{r}) \varphi_{a}^{*}(\mathbf{r}) f_{\mathrm{Mxc}}^{q_{\alpha}}\left(\mathbf{r}, \Omega_{q}\right) \\
& N_{\alpha, i a}=\frac{1}{2 \omega_{\alpha}^{2}} \int \operatorname{d} \mathbf{r} \varphi_{i}^{*}(\mathbf{r}) \varphi_{a}(\mathbf{r}) g_{M}^{n_{\alpha}}(\mathbf{r})
\end{aligned}
$$

We emphasize here that the exact coupling matrix $N_{\alpha, i a}$ has no frequency dependence since the exact kernel for eq 42 is equivalent to just the mean-field kernel of the photon modes, as can be seen from eq 9. Given the exact kernels, the nonlinear pseudoeigenvalue problem in eq 39 allows to compute the exact excitation energies of the coupled matter-photon system. Of course, in practice, approximations have to be employed for the matter-photon response kernels, as is also required in the matter-only response formalism. Since the explicitly known mean-field kernel $g_{M}^{n_{\alpha}}(\mathbf{r})$ is already exact, only $f_{\mathrm{Mxc}}^{q_{\alpha}}\left(\mathbf{r}, \Omega_{q}\right)$ and $f_{\mathrm{Mxc}}^{n}\left(\mathbf{r}, \mathbf{y}, \Omega_{q}\right)$ are left to be approximated.

The above matrix equation of eq 39 can be cast into a Hermitian eigenvalue form following the same transformations as, for example, in ref 90 , where we assume real-valued orbitals, that is, $K=K^{*}, M=M^{*}$ and $N=N^{*}$. Further, we drop the dependency on $\Omega_{q}$ for brevity. Then we find the pseudoeigenvalue equation, reminiscent to the equations found for excitation energies in Hartree-Fock theory and TDDFT. $^{65}$ The eigenvalue problem of eq 39 is now written in a compact Hermitian form as

$$
\left(\begin{array}{cc}
U & V \\
V^{\dagger} & \omega_{\alpha}^{2}
\end{array}\right)\left(\begin{array}{l}
\mathbf{E}_{1} \\
\mathbf{P}_{1}
\end{array}\right)=\Omega_{q}^{2}\left(\begin{array}{c}
\mathbf{E}_{1} \\
\mathbf{P}_{1}
\end{array}\right)
$$

where the matrices $U$ and $V$ are given by $U=(L-K)^{1 / 2}(L+$ K) $(L-K)^{1 / 2}, V=2(L-K)^{1 / 2} M^{1 / 2} N^{1 / 2} \omega_{\alpha}^{1 / 2}$ and the matrices are given explicitly by

$$
\begin{aligned}
& U_{q q^{\prime}}=\delta_{q q^{\prime}} \omega_{q}^{2}+2 \sqrt{\omega_{q} \omega_{q^{\prime}}} K_{q q^{\prime}}\left(\Omega_{q}\right) \\
& V_{q \alpha}=2 \sqrt{\omega_{q} M_{\alpha q}\left(\Omega_{q}\right) N_{\alpha q} \omega_{\alpha}}
\end{aligned}
$$

The index $q=(a, i)$ describes transitions from the electronic occupied $(i)$ to unoccupied states $(a)$, and thus, the difference of Kohn-Sham energies is given by $\omega_{q}=\epsilon_{a}-\epsilon_{i}$. With $\alpha$ we denote the photon modes. The eigenvectors $\mathbf{E}_{1}$ and $\mathbf{P}_{1}$ can be used to compute oscillator strengths of the coupled matterphoton system (see SI, S5). In the decoupling limit of the light-matter interaction, eq 43 reduces to the well-known
Casida equation (eq S4 in the SI). ${ }^{65}$ So far we did not solve anything but have just rewritten the problem in terms of unknown Mxc kernels that correct the uncoupled and noninteracting auxiliary response functions. To actually solve this problem we need to provide approximations to these unknown quantities. Here it becomes advantageous to have divided the full Mxc kernels in Hxc and pxc terms, such that we can use well-established approximations from electronic TDDFT for the Hxc and specifically developed approximations for the pxc terms (see section 2 for more details). In the following, we will employ the above introduced pRPA approximation, which is a straightforward generalization of the standard RPA of electronic-structure theory and yields the following kernels

$$
\begin{aligned}
& f_{\mathrm{H}}^{n}\left(\mathbf{r}, \mathbf{r}^{\prime}\right)=\frac{e^{2}}{4 \pi \epsilon_{0}\left|\mathbf{r}-\mathbf{r}^{\prime}\right|}, f_{\mathrm{p}}^{q_{\alpha}}(\mathbf{r})=-\omega_{\alpha} \lambda_{\alpha} \cdot e \mathbf{r}, \\
& f_{\mathrm{p}}^{n_{\alpha}}\left(\mathbf{r}, \mathbf{r}^{\prime}\right)=\sum_{\alpha}\left(\lambda_{\alpha} \cdot e \mathbf{r}^{\prime}\right) \lambda_{\alpha} \cdot e \mathbf{r}, g_{\mathrm{M}}^{n_{\alpha}}(\mathbf{r})=-\omega_{\alpha}^{2} \lambda_{\alpha} \cdot e \mathbf{r}
\end{aligned}
$$

We note that, at the pRPA level, the matter-photon coupling mediated via $g_{M}^{n_{\alpha}}$ is exact. The influence of the photon-matter $\mathrm{xc}$ contributions, $f_{\mathrm{xc}}^{q_{\alpha}}$ and $f_{\mathrm{xc}}^{n}$, will be highlighted in the next section. By connecting to the eigenstates $\mathbf{E}_{1}$ and $\mathbf{P}_{1}$, we can assign to each of the individual poles of the response function, that is, the excitation energies, the amount of photonic and electronic contribution to that excitation by using

$$
\begin{aligned}
& \sigma_{e}=\sum_{i=1}^{N_{\text {pars }}}\left|E_{1, i}\right|^{2} \\
& \sigma_{p}=\sum_{\alpha=1}^{M}\left|P_{1, \alpha}\right|^{2}
\end{aligned}
$$

where $N_{\text {pairs }}$ corresponds to the number of occupiedunoccupied pairs of KS orbitals, in our case, $30 \times 500$. The sum of $\sigma_{e}$ and $\sigma_{p}$ is normalized to one, that is, $\sigma_{e}+\sigma_{p}=1$.

In the pRPA approximation, all the frequency dependence that we suppressed at times for brevity now genuinely vanishes (an adiabatic approximation), which allows us to express $M$ and $N$ of eqs 41 and 42 as

$$
\begin{aligned}
& M_{\alpha, a i}=-\omega_{\alpha} \int \mathrm{d} r \varphi_{i}(\mathbf{r}) \varphi_{a}^{*}(\mathbf{r}) \lambda_{\alpha} \cdot e \mathbf{r} \\
& N_{\alpha, i a}=-\frac{1}{2} \int \mathrm{d} r \varphi_{i}^{*}(\mathbf{r}) \varphi_{a}(\mathbf{r}) \lambda_{\alpha} \cdot e \mathbf{r}
\end{aligned}
$$

Next, we want to connect to the standard matter-only linearresponse framework. ${ }^{59}$ In defining the oscillator strength for the density-density response function, we make use of the relationship between the polarizability tensor and susceptibility. The first-order dipole polarizability is given by

$$
\delta \mathbf{R}(t)=\int \mathrm{d} \mathbf{r} e \mathbf{r} \delta n(\mathbf{r}, t)
$$

and in frequency space $\mathbf{R}(\omega)=\overleftrightarrow{\alpha}(\omega) \mathbf{E}(\omega)$. The dynamic polarizability tensor can then be written as

$$
\overleftrightarrow{\alpha_{\mu \nu}}(\omega)=\int \mathrm{d} \mathbf{r} e r_{\mu} \frac{\delta n(\mathbf{r}, \omega)}{\delta E_{\nu}(\omega)}
$$

with $\mu, \nu=1,2$, and 3, denoting all three spatial directions. Connecting to the QEDFT linear-response theory, we find 


$$
\overleftrightarrow{\alpha}_{\mu \nu}(\omega)=\sum_{I} \frac{2 \mathbf{r}_{\mu}^{\dagger} S^{1 / 2} \mathbf{Z}_{I} \mathbf{Z}_{I}^{\dagger} S^{1 / 2} \mathbf{r}_{\nu}}{\omega^{2}-\Omega_{I}^{2}}
$$

where $\mathbf{r}_{\mu}^{I}=\int \mathrm{d} \mathbf{r} e r_{\mu} \sum_{i, a} \Phi_{i a}(\mathbf{r})$ is the Kohn-Sham transition dipole matrix element of the many-body transition $I$. Further, we have used $S=(L-K)$, and the transition density is defined as $\Phi_{i a}(\mathbf{r})=\varphi_{i}^{*}(\mathbf{r}) \varphi_{a}(\mathbf{r})$ in terms of Kohn-Sham orbitals. This then allows to obtain the full photoabsorption cross section from the trace of the polarizability tensor through

$$
\sigma(\omega)=\frac{4 \pi \omega}{c} \operatorname{Im} \operatorname{Tr} \overleftrightarrow{\alpha}(\omega) / 3
$$

For the oscillator strength, ${ }^{59,65}$ we find

$$
f_{I}=\frac{2}{3} \sum_{\mu=1}^{3}\left|\mathbf{Z}_{I}^{\dagger} S^{1 / 2} \mathbf{r}_{\mu}^{I}\right|^{2}=\frac{2}{3} \omega_{I} \sum_{\mu=1}^{3}\left|\left\langle\Psi_{0}\left|e r_{\mu}\right| \Psi_{I}\right\rangle\right|^{2}
$$

and also, in the case of QEDFT, the oscillator strength satisfy the Thomas-Reiche-Kuhn sum rule (also known as $f$-sum rule), that is, $\sum_{I} f_{I}=N$, where $N$ is the total number of electrons in the system. At this point, we also want to introduce the dipole strength function $S(\omega)^{59}$ that is defined as

$$
S(\omega)=\sum_{I} f_{I} \delta\left(\omega-\Omega_{I}\right)
$$

and integrates according to the $f$-sum rule to the total number of electrons. For the nonstandard part of our response theory, that is, matter-photon and photon-photon perturbations, we use similar constructions to display the results. Their derivations and definitions are given in SI S5. We will discuss their physical meaning in the next section, where we employ a simple yet illuminating model system. This will not only allow us to explain many of the so far abstract ideas in a straightforward manner, but we can also test the accuracy of the pRPA.

\section{EXAMPLES FOR THE COUPLED MATTER-PHOTON RESPONSE: DETAILS ON THE RABI MODEL}

In this section, we give more details on the model system that has been employed in section 2. The model Hamiltonian we consider is given by (in this section, we switch for simplicity to atomic units)

$$
\hat{H}_{R}(t)=\frac{\omega_{0}}{2} \hat{\sigma}_{z}+\omega_{c} \hat{c}^{\dagger} \hat{a}+\lambda \hat{\sigma}_{x} \hat{q}+j(t) \hat{q}+v(t) \hat{\sigma}_{x}
$$

where $\omega_{0}$ is the transition frequency between the ground state I $g\rangle$ and excited state $|e\rangle$ and $\hat{\sigma}_{x}$ as well as $\hat{\sigma}_{z}$ are the usual Pauli matrices. We only keep one photon mode with frequency $\omega_{c}$ and use the usual photon creation and annihilation operators to represent the harmonic oscillator of this mode. By further compressing the notation, we then describe the coupling between matter and light by a coupling strength $\lambda$ and the displacement coordinate $\hat{q}=\frac{1}{\sqrt{2 \omega_{c}}}\left(\hat{a}+\hat{a}^{\dagger}\right)$. Finally, we couple the matter system to a classical external perturbation $v(t)$ and the photon system to a classical external current $j(t)$ (a pictorial representation of the coupled system is given in Figure 3). We note for consistency with respect to other works $^{37,50,52}$ that, in the above Rabi model, we can perform a unitary transformation that allows us to exchange $\hat{\sigma}_{x}$ and $\hat{\sigma}_{z}$. Both forms of the extended Rabi model are therefore equivalent. We further note that, with respect to the full nonrelativistic QED problem in the long wavelength approximation of eq 1 , the Rabi model does not include the dipole self-energy term proportional to $(\lambda \cdot \mathbf{R})^{2}$. This is because the analogous term in this model is just a constant energy shift, that is, it is proportional to $\sigma_{x}^{2}=\hat{\mathbb{l}} .^{47}$ For more levels, this is no longer the case, ${ }^{52}$ and this term has to be taken into account or else the resulting eigenstates do not have a proper continuum limit. ${ }^{47}$ The responses that we want to consider in the following are those observables that couple to the external perturbations. In our case, this is $\sigma_{x}(t)=\left\langle\Psi(t)\left|\hat{\sigma}_{x}\right| \Psi(t)\right\rangle$ (in essence the atomic dipole) and the displacement field $q(t)=$ $\langle\Psi(t)|\hat{q}| \Psi(t)\rangle$.

The response of these observables $\left(\delta \sigma_{x}(t), \delta q(t)\right)$ to perturbations by the external pair $(\delta v(t), \delta j(t))$ can be written similarly as eq 28 in the collective form

$$
\left(\begin{array}{c}
\delta \sigma_{x}(t) \\
\delta q(t)
\end{array}\right)=\int d t^{\prime}\left(\begin{array}{cc}
\chi_{\sigma_{x}}^{\sigma_{x}}\left(t, t^{\prime}\right) & \chi_{q}^{\sigma_{x}}\left(t, t^{\prime}\right) \\
\chi_{\sigma_{x}}^{q}\left(t, t^{\prime}\right) & \chi_{q}^{q}\left(t, t^{\prime}\right)
\end{array}\right)\left(\begin{array}{c}
\delta v\left(t^{\prime}\right) \\
\delta j\left(t^{\prime}\right)
\end{array}\right)
$$

Again, we find besides the usual matter-matter response, $\chi_{\sigma_{x}}^{\sigma_{x}}$ also matter-photon responses $\chi_{q}^{\sigma_{x}}$ and $\chi_{\sigma_{x}}^{q}$, respectively, as well as a photon-photon response function $\chi_{q}^{q}$. Next, in analogy to section 1 , we reformulate the coupled matter-photon problem in form of a Maxwell KS auxiliary problem. Using by now wellestablished results of QEDFT for the extended Rabi model systems, ${ }^{37,50}$ we can introduce two effective fields

$$
\begin{aligned}
& v_{\mathrm{Mxc}}\left(\left[\sigma_{x}, q\right] ; t\right)=v_{s}\left(\left[\sigma_{x}\right] ; t\right)-v\left(\left[\sigma_{x}, q\right] ; t\right) \\
& j_{\mathrm{M}}\left(\left[\sigma_{x}\right] ; t\right)=j_{s}([q] ; t)-j\left(\left[\sigma_{x}, q\right] ; t\right)
\end{aligned}
$$

that force the auxiliary uncoupled, yet nonlinear Maxwell KS system to generate the same dynamics of the internal pair $\left(\sigma_{x}(t), q(t)\right)$ as the corresponding coupled reference system. For an uncoupled initial Maxwell state $\left|\Psi_{0}\right\rangle=\left|\psi_{0}\right\rangle \otimes\left|\varphi_{0}\right\rangle$ that provides the same initial conditions for the internal pair as the physical initial state, ${ }^{37,50}$ we then have to solve self-consistently

$$
\begin{aligned}
& i \frac{\partial}{\partial t}|\psi(t)\rangle=\left[\frac{\omega_{0}}{2} \hat{\sigma}_{z}+\left(v(t)+v_{\mathrm{Mxc}}\left(\left[\sigma_{x}, q\right] ; t\right)\right) \hat{\sigma}_{x}\right]|\psi(t)\rangle \\
& \left(\frac{\partial^{2}}{\partial t^{2}}+\omega_{c}^{2}\right) q(t)=-j(t)-\lambda \sigma_{x}(t)
\end{aligned}
$$

Since the photon subsystem is merely a shifted harmonic oscillator we get away with only solving the classical harmonic oscillator equation coupled to the dipole of the matter subsystem. We can then express the coupled response functions of eq 57 in analogy to eqs $10-13$ by the uncoupled auxiliary response functions $\chi_{\sigma_{x}, s}^{\sigma_{x}}$ and $\chi_{q, s}^{q}$ as

$$
\begin{aligned}
\chi_{\sigma_{x}}^{\sigma_{x}}\left(t, t^{\prime}\right)= & \chi_{\sigma_{x}, s}^{\sigma_{x}}\left(t, t^{\prime}\right)+\iint \mathrm{d} \tau \mathrm{d} \tau^{\prime} \chi_{\sigma_{x}, s}^{\sigma_{x}}(t, \tau) f_{\mathrm{Mxc}}^{\sigma_{x}}\left(\tau, \tau^{\prime}\right) \chi_{\sigma_{x}}^{\sigma_{x}}\left(\tau^{\prime}, t^{\prime}\right) \\
& +\iint \mathrm{d} \tau \mathrm{d} \tau^{\prime} \chi_{\sigma_{x}, s}^{\sigma_{x}}(t, \tau) f_{\mathrm{Mxc}}^{q}\left(\tau, \tau^{\prime}\right) \chi_{\sigma_{x}}^{q}\left(\tau^{\prime}, t^{\prime}\right) \\
\chi_{q}^{\sigma_{x}}\left(t, t^{\prime}\right)= & \iint \mathrm{d} \tau \mathrm{d} \tau^{\prime} \chi_{\sigma_{x}, s}^{\sigma_{x}}(t, \tau) f_{\mathrm{Mxc}}^{q}\left(\tau, \tau^{\prime}\right) \chi_{q}^{q}\left(\tau^{\prime}, t^{\prime}\right) \\
& +\iint \mathrm{d} \tau \mathrm{d} \tau^{\prime} \chi_{\sigma_{x}, s}^{\sigma_{x}}(t, \tau) f_{\mathrm{Mxc}}^{\sigma_{x}}\left(\tau, \tau^{\prime}\right) \chi_{q}^{\sigma_{x}}\left(\tau^{\prime}, t^{\prime}\right) \\
\chi_{\sigma_{x}}^{q}\left(t, t^{\prime}\right)= & \iint \mathrm{d} \tau \mathrm{d} \tau^{\prime} \chi_{q, s}^{q}(t, \tau) g_{M}^{\sigma_{x}}\left(\tau, \tau^{\prime}\right) \chi_{\sigma_{x}}^{\sigma_{x}}\left(\tau^{\prime}, t^{\prime}\right) \\
\chi_{q}^{q}\left(t, t^{\prime}\right)= & \chi_{q, s}^{q}\left(t, t^{\prime}\right)+\iint \mathrm{d} \tau \mathrm{d} \tau^{\prime} \chi_{q, s}^{q}(t, \tau) g_{M}^{\sigma_{x}}\left(\tau, \tau^{\prime}\right) \chi_{q}^{\sigma_{x}}\left(\tau^{\prime}, t^{\prime}\right)
\end{aligned}
$$


The only real difference is that in the Rabi case we do not have a longitudinal interaction and therefore the Mxc contributions come solely from the matter-photon coupling, that is, $f_{\mathrm{Mxc}}^{\sigma_{x}}=$ $f_{\mathrm{pxc}}^{\sigma_{x}}$ and $f_{\mathrm{Mxc}}^{q}=f_{\mathrm{pxc}}^{q}$. This allows us to study exclusively the influence of these new terms and how approximations of them perform.

Matter-Photon Correlation Effect in Maxwell's Equations

Let us follow the previous general section 1.4 and briefly consider the influence of the matter-photon coupling on the Maxwell's equations in this model system, that is, eq 61 . The inhomogeneous Maxwell's equation here accounts for the back-reaction of the matter on the field through the atomic dipole operator $\sigma_{x}([v, j] ; t)$. If we, for instance, perturb the two-level system directly via a $\delta v(t)$, the response of the Maxwell's equation expressed in terms of the uncoupled problem with the help of eq 63 becomes

$$
\begin{aligned}
& \left(\partial_{t}^{2}+\omega_{c}^{2}\right) \delta q(t)=-\lambda \int \mathrm{d} t^{\prime} \chi_{\sigma_{x}, s}^{\sigma_{x}}\left(t, t^{\prime}\right) \delta v\left(t^{\prime}\right) \\
& \quad-\lambda \iiint \mathrm{d} t^{\prime} \mathrm{d} \tau \mathrm{d} \tau^{\prime} \chi_{\sigma_{x}, s}^{\sigma_{x}}(t, \tau) f_{\mathrm{Mxc}}^{\sigma_{x}}\left(\tau, \tau^{\prime}\right) \chi_{\sigma_{x}}^{\sigma_{x}}\left(\tau^{\prime}, t^{\prime}\right) \delta v\left(t^{\prime}\right) \\
& \quad-\lambda \iint \mathrm{d} \tau \mathrm{d} \tau^{\prime} \chi_{\sigma_{x}, s}^{\sigma_{x}}(t, \tau) f_{\mathrm{Mxc}}^{q}\left(\tau, \tau^{\prime}\right) \delta q\left(\tau^{\prime}\right)
\end{aligned}
$$

Having no coupling, that is, the Mxc terms are zero, merely recovers the usual inhomogeneous Maxwell's equation for a classical external current. The matter system evolves according to the perturbation and we can determine its induced Maxwell field without any back-reaction. The second term describes the matter polarization due to the induced field and leads to an effective self-interaction of the two-level system. If there was more than one particle, this would induce an effective mattermatter interaction as well. The third term then accounts for the field polarization and induces an effective self-interaction in the mode of the light field. That is, the coupling to matter leads to a photon-photon interaction. This can be made more explicit by separating the mean-field contribution $v_{M}(t)=\lambda q(t)$ and rewriting the above equation as

$$
\begin{aligned}
& \left(\partial_{t}^{2}+\omega_{c}^{2}\right) \delta q(t)=-\lambda \int \mathrm{d} t^{\prime} \chi_{\sigma_{x}, s}^{\sigma_{x}}\left(t, t^{\prime}\right) \delta v\left(t^{\prime}\right) \\
& \quad-\lambda \iiint \mathrm{d} t^{\prime} \mathrm{d} \tau \mathrm{d} \tau^{\prime} \chi_{\sigma_{x}, s}^{\sigma_{x}}(t, \tau) f_{\mathrm{xc}}^{\sigma_{x}}\left(\tau, \tau^{\prime}\right) \chi_{\sigma_{x}}^{\sigma_{x}}\left(\tau^{\prime}, t^{\prime}\right) \delta v\left(t^{\prime}\right) \\
& \quad-\lambda^{2} \int \mathrm{d} \tau \chi_{\sigma_{x}, s}^{\sigma_{x}}(t, \tau) \delta q(\tau)-\lambda \iint \mathrm{d} \tau \mathrm{d} \tau^{\prime} \chi_{\sigma_{x}, s}^{\sigma_{x}}(t, \tau) f_{\mathrm{xc}}^{q}\left(\tau, \tau^{\prime}\right) \delta q\left(\tau^{\prime}\right)
\end{aligned}
$$

The third term on the right-hand side is then the pRPA form of photon-photon response. Similar terms also appear for a perturbation induced by an external current $\delta j(t)$, which can be rewritten with the help of eq 64 and the mean-field made explicit as

$$
\begin{aligned}
& \left(\partial_{t}^{2}+\omega_{c}^{2}\right) \delta q(t)=-\delta j(t)-\lambda^{2} \int \mathrm{d} \tau \chi_{\sigma_{x}, s}^{\sigma_{x}}(t, \tau) \delta q(\tau) \\
& \quad-\lambda \iint \mathrm{d} \tau \mathrm{d} \tau^{\prime} \chi_{\sigma_{x}, s}^{\sigma_{x}}(t, \tau) f_{\mathrm{xc}}^{q}\left(\tau, \tau^{\prime}\right) \delta q\left(\tau^{\prime}\right) \\
& \quad-\lambda \iiint \mathrm{d} t^{\prime} \mathrm{d} \tau \mathrm{d} \tau^{\prime} \chi_{\sigma_{x}, s}^{\sigma_{x}}(t, \tau) f_{\mathrm{xc}}^{\sigma_{x}}\left(\tau, \tau^{\prime}\right) \chi_{q}^{\sigma_{x}}\left(\tau^{\prime}, t^{\prime}\right) \delta j\left(t^{\prime}\right)
\end{aligned}
$$

Here we used that $f_{M}^{q}\left(\tau, \tau^{\prime}\right)=\lambda \delta\left(\tau-\tau^{\prime}\right)$. As is most obvious in the pRPA limit, both types of perturbations lead to the same resonance conditions, i.e., peaks in the responses. They are connected to the combined eigenstates of the matter-photon system. However, the detailed response can differ strongly. That these resonance conditions that we get from the pRPA are indeed connected to the coupled eigenstates, we will show next.

\section{Application of the Pseudo-Eigenvalue Problem}

As a preparatory step, we first rewrite the linear-response problem of the extended Rabi model in terms of the previously introduced pseudo-eigenvalue problem of eq 43. In the twolevel one-mode case we consider here, this reduces to

$$
\left(\begin{array}{cc}
U\left(\Omega_{q}\right) & V\left(\Omega_{q}\right) \\
V^{*}\left(\Omega_{q}\right) & \omega_{c}^{2}
\end{array}\right)\left(\begin{array}{c}
\mathbf{E}_{1} \\
\mathbf{P}_{1}
\end{array}\right)=\Omega_{q}^{2}\left(\begin{array}{c}
\mathbf{E}_{1} \\
\mathbf{P}_{1}
\end{array}\right)
$$

where the matrices in the model system reduce to functions of $\Omega_{q}$ as $U=\omega_{0}^{2}+2 \omega_{0} K\left(\Omega_{q}\right), V\left(\Omega_{q}\right)=2 \omega_{0}^{1 / 2} M\left(\Omega_{q}\right)^{1 / 2} N^{1 / 2} \omega_{c}^{1 / 2}$, and $V^{*}\left(\Omega_{q}\right)=2 \omega_{c}^{1 / 2} N^{1 / 2} M\left(\Omega_{q}\right)^{1 / 2} \omega_{0}^{1 / 2}$. The coupling functions are given explicitly using eqs $(40-(42)$ as

$$
\begin{aligned}
K\left(\Omega_{q}\right) & =f_{M}^{\sigma_{x}}+f_{x c}^{\sigma_{x}}\left(\Omega_{q}\right), \\
M\left(\Omega_{q}\right) & =f_{M}^{q}+f_{x c}^{q}\left(\Omega_{q}\right), \\
N & =\frac{1}{2 \omega_{c}} g_{M}^{\sigma_{x}}
\end{aligned}
$$

where the Kohn-Sham states is the dipole matrix element $\varphi_{a} \varphi_{i}^{*}$ $=\left\langle g\left|\hat{\sigma}_{x}\right| e\right\rangle=1$. The Mxc kernels can be defined using the inverse of the auxiliary and interacting response functions (see also eqs S30 and S31 in the SI) and are given in the frequency space by

$$
\begin{aligned}
& f_{\mathrm{Mxc}}^{\sigma_{x}}(\omega)=\left(\chi_{\sigma_{x}, s}^{\sigma_{x}}(\omega)\right)^{-1}-\left(\chi_{\sigma_{x}}^{\sigma_{x}}(\omega)\right)^{-1} \\
& f_{\mathrm{Mxc}}^{q}(\omega)=-\left(\chi_{n}^{q}(\omega)\right)^{-1}
\end{aligned}
$$

Here, $\left(\chi_{\sigma_{x} s}^{\sigma_{x}}(\omega)\right)^{-1},\left(\chi_{\sigma_{x}}^{\sigma_{x}}(\omega)\right)^{-1}$, and $\left(\chi_{n}^{q}(\omega)\right)^{-1}$ are the inverses of the uncoupled response function of the electronic subsystem and the fully coupled response function of the electronic dipole and of the displacement field of the Rabi model, respectively. With these quantities, we then determine spectroscopic observables such as the photoabsorption cross section. To determine this cross section we first note that the linear polarizability $\alpha(\omega)$ induced by the external potential $v(\omega)$ is related to the "dipole-dipole" response function as $\alpha(\omega)=$ $\chi_{\sigma_{x}}^{\sigma_{x}}(\omega)$. Using eq 53, we can determine the photoabsorption cross section of the Rabi model (see Figure 4a displayed in dotted-red for the numerically exact case).

$$
\sigma(\omega)=\frac{4 \pi \omega}{c} \mathcal{I} m \chi_{\sigma_{x}}^{\sigma_{x}}(\omega)
$$

Here, the mean of the polarizability was not considered since the Rabi model is a one-dimensional system. Analogously, we define a linear "field polarizability" $\beta(\omega)$ due to polarizing the photon mode by an external current. In the same way, we relate the field polarizability to the response function of the photon mode as $\beta(\omega)=\chi_{q}^{q}(\omega)$ and then determine a photonic spectrum from (see Figure $4 \mathrm{~b}$ displayed in dotted-red for the numerically exact case).

$$
\tilde{\sigma}(\omega) \equiv \frac{4 \pi \omega}{c} \mathcal{I} m \chi_{q}^{q}(\omega)
$$

Finally, we consider mixed spectroscopic observables where we perturb one subsystem and then consider the response in the other. We analogously employ $\chi_{\sigma_{x}}^{q}(\omega)$ and $\chi_{q}^{\sigma_{x}}(\omega)$ in eqs 72 and 73 , respectively, to determine a "mixed polarizability". If we plot this mixed spectrum (see Figure $4 \mathrm{c}$ displayed in dotted- 
red for the numerically exact case), we find that we have positive and negative peaks. Indeed, this highlights that excitations due to external perturbations can be exchanged between subsystems, that is, energy absorbed in the electronic subsystem can excite the photonic subsystem and vice versa. Next, we want to employ the pRPA approximation to the extended Rabi model and try to solve it analytically. The pRPA is equivalent to using the mean-field approximation in the coupled equations, that is, approximating the electron-photon coupling term as $\hat{\sigma}_{x} \hat{q} \approx\left\langle\hat{\sigma}_{x}\right\rangle \hat{q}+\langle\hat{q}\rangle \hat{\sigma}_{x}$. This corresponds then to a coupled Schrödinger-Maxwell treatment of the coupled matter-photon problem. ${ }^{13}$ In the Maxwell KS equations this leads to approximating the full $v_{\mathrm{Mxc}}$ by the mean-field potential $v_{\mathrm{M}}=v_{\mathrm{p}}=\lambda q$. The mean-field current is known explicitly as $j_{\mathrm{M}}=$ $\lambda \sigma_{x}$. In the case of the pseudo-eigenvalue problem this amounts to approximating $K=f_{M}^{\sigma_{x}}=0, \quad M=f_{M}^{q}=\lambda$ and $N=\frac{1}{2 \omega_{\mathrm{c}}}, g_{\mathrm{M}}^{\sigma_{x}}=\frac{\lambda}{2 \omega_{\mathrm{c}}}$. Consequently, we have

$$
U=\omega_{0}^{2}, \quad V=W=2 \lambda \sqrt{\frac{\omega_{0}}{2}}, \quad \omega_{\alpha}^{2}=\omega_{c}^{2}
$$

The resulting nonlinear eigenvalue equation yields the excitation frequencies

$$
\begin{aligned}
& \Omega_{1}^{2}(-)=\frac{1}{2}\left(\omega_{0}^{2}+\omega_{c}^{2}\right)-\frac{1}{2} \sqrt{\left(\omega_{0}^{2}-\omega_{c}^{2}\right)^{2}+8 \lambda^{2} \omega_{0}} \\
& \Omega_{1}^{2}(+)=\frac{1}{2}\left(\omega_{0}^{2}+\omega_{c}^{2}\right)+\frac{1}{2} \sqrt{\left(\omega_{0}^{2}-\omega_{c}^{2}\right)^{2}+8 \lambda^{2} \omega_{0}}
\end{aligned}
$$

and the corresponding normalized eigenvectors can be given in closed form as

$$
\mathbf{E}_{1}=\left(\begin{array}{c}
-\sin \theta \\
\cos \theta
\end{array}\right) \text { and } \mathbf{P}_{1}=\left(\begin{array}{c}
\cos \theta \\
\sin \theta
\end{array}\right)
$$

The resulting pRPA-approximated spectra are displayed in Figure 4 in dashed-blue. We will discuss the results in a little more detail at the end of this section, before we consider a slightly more advanced approximation based on the rotatingwave approximation (RWA). If we slightly simplify the full Rabi problem by approximating the full coupling as $\hat{\sigma}_{x} \hat{q} \approx \frac{1}{\sqrt{2 \omega_{c}}}\left(\hat{\sigma}_{+} \hat{a}+\hat{\sigma}_{-} \hat{a}^{\dagger}\right)$, we end up with the Jaynes-Cumming Hamiltonian $^{91}$ given as

$$
\hat{H}_{\mathrm{JC}}(t)=\frac{\omega_{0}}{2} \hat{\sigma}_{z}+\omega_{c} \hat{a}^{\dagger} \hat{a}+\frac{\lambda}{\sqrt{2 \omega_{\mathrm{c}}}}\left(\hat{\sigma}_{+} \hat{a}+\hat{\sigma}_{-} \hat{a}^{\dagger}\right)+j(t) \hat{q}+v(t) \hat{\sigma}_{x}
$$

Here we used $\hat{\sigma}_{ \pm}=\left(\hat{\sigma}_{x} \pm i \hat{\sigma}_{y}\right) / 2$. The above approximation is called the RWA because we ignore quickly oscillating terms and thus assume that the excitation of the matter subsystem can only destroy and the de-excitation only create a photon. This approximation is justified (with respect to the full wave function) if we are in the weak coupling regime, that is, $\lambda \ll$ $\omega_{\mathrm{c}}$, and near to resonance, that is, $\delta=\omega_{0}-\omega_{\mathrm{c}} \approx 0$. The ground-state of the Jaynes-Cummings model is the uncoupled tensor product of the matter ground-state and the photon ground-state with ground-state energy of $E_{0}=-\omega_{0} / 2$. The excited states of the Jaynes-Cummings Hamiltonian are known analytically and are given by (we only show the lowest lying excited states where a single photon is excited and for which the matrix elements are non-zero with the ground-state)

$$
\begin{aligned}
& |-, 0\rangle=-\sin \theta_{0}|g\rangle|1\rangle+\cos \theta_{0}|e\rangle|0\rangle \\
& |+, 0\rangle=\cos \theta_{0}|g\rangle|1\rangle+\sin \theta_{0}|e\rangle|0\rangle
\end{aligned}
$$

With these eigenstates, we find the transition frequencies that correspond to the linear response from the ground state (due to the approximations involved only one photon absorbed or emitted) to be

$$
\begin{aligned}
& \Omega_{-}(0)=\frac{1}{2}\left(\omega_{c}+\omega_{0}-\Omega_{0}\right) \\
& \Omega_{+}(0)=\frac{1}{2}\left(\omega_{c}+\omega_{0}+\Omega_{0}\right)
\end{aligned}
$$

where $\Omega_{0}=\sqrt{\delta^{2}+4 \lambda^{\prime 2}}$ where $\lambda^{\prime}=\frac{\lambda}{\sqrt{2 \omega_{c}}}$. So we already know where the RWA will generate the poles of the response function. Since we know analytically the eigenfunctions in the RWA, we can construct the RWA response functions analytically. Using the definitions of the Mxc kernels of eq 70 we can then analytically construct the RWA Mxc kernels. These kernels are frequency dependent, therefore the resulting Mxc approximation is non-adiabatic. ${ }^{59}$ Substituting them into $M\left(\Omega_{q}\right)$ we recover the known poles $\Omega_{q}=\Omega_{ \pm}(0)$ from eq 69 . Further, we can then construct the different spectra associated with the RWA. We show them in Figure 4 in full-orange.

\section{NUMERICAL DETAILS}

We start by discussing the general setup before considering the specialized situations discussed above. We have implemented the linear-response pseudoeigenvalue equation of eq 43 into the real-space code OCTOPUS. ${ }^{76,77}$ The absorption spectrum of the benzene molecule has been very successfully studied with TDDFT calculations. ${ }^{76,78}$ Small organic molecules and benzene in particular are rewarding systems to be studied with TDDFT, since the adiabatic approximation in concert with the local-density approximation (LDA) ${ }^{92,93}$ capture the occurring $\Pi-\Pi^{*}$ transition exceptionally well. ${ }^{78}$ This transition is a characteristic of carbon conjugate compounds ${ }^{76}$ and occurs around $7 \mathrm{eV}$ in the case of a benzene molecule. To calculate the electronic structure of the benzene molecule, we follow closely the setup of ref 76 . Thus, we use a cylindric real space grid of $8 \AA$ length with the radius of $6 \AA$ in the $x-y$ plane and a spacing of $\Delta x=0.22 \AA$. For the benzene nuclear structure, we use the $\mathrm{C}-\mathrm{C}$ bond length of $1.396 \AA$ and $\mathrm{C}-\mathrm{H}$ bond length of $1.083 \AA$. We explicitly describe the 30 valence electrons, while the core atoms are considered implicitly by LDA TroullierMartins pseudopotentials. ${ }^{94}$ In the excited state manifold, we include 500 unoccupied states in the pseudoeigenvalue calculation. This number amounts to $30 \times 500=7500$ pairs of occupied-unoccupied states. Further, to describe the electron-electron interaction in the response functions, we apply the adiabatic LDA (ALDA) kernel, that is, $f_{\mathrm{Mxc}}^{n} \rightarrow$ $f_{\text {Hxc,ALDA }}^{n}+f_{p}^{n}$. Solving the linear-response pseudoeigenvalue problem of eq 43 provides us with the transition amplitudes, as well as the excitation energies of the correlated electronphoton system. These quantities can be used to calculate photoabsorption spectra by using, for example, eq 55. In standard calculations, to obtain such spectra and mimic the finite lifetime of the excited state usually a peak-broadening is applied. In our case, where necessary, we apply a Lorentzian broadening, that is, the standard implementation of the OCTOPUS code, that is of the following form 


$$
\Gamma\left(\omega, \omega_{I}\right)=\frac{1}{\pi} \frac{\Delta}{\left(\omega-\omega_{I}\right)^{2}+\Delta^{2}}
$$

where $\omega_{I}$ is the excitation frequency, and $\Delta$ is the broadening parameter. The actual dipole strength function as defined in eq 55 is then obtained by

$$
S(\omega)=\sum_{I} f_{I} \Gamma\left(\omega, \omega_{I}\right)
$$

where $f_{I}$ denotes the oscillator strength as defined in eq 54 . We obtain the spectra for systems not immersed in the photon bath in this paper, where the peaks have been broadened by applying the broadening as defined in eq 82 with $\Delta=0.1361$ $\mathrm{eV}$.

\section{ASSOCIATED CONTENT}

\section{S Supporting Information}

The Supporting Information is available free of charge on the ACS Publications website at DOI: 10.1021/acsphotonics.9b00768.

Additional information about the current state of the art in spectroscopy, that is, the semiclassical description of light-matter coupled systems. In addition, we provide detailed derivations on linear-response theory in nonrelativistic QED, within QEDFT, and the matrix formulations of the QEDFT response equations. We further derive the oscillator strengths for the photonmatter, matter-photon, and the photon-photon response functions (PDF)

\section{AUTHOR INFORMATION}

\section{Corresponding Authors}

*E-mail: jflick@flatironinstitute.org.

*E-mail: davis.welakuh@mpsd.mpg.de.

*E-mail: michael.ruggenthaler@mpsd.mpg.de.

*E-mail: heiko.appel@mpsd.mpg.de.

*E-mail: angel.rubio@mpsd.mpg.de.

\section{ORCID $\odot$}

Johannes Flick: 0000-0003-0273-7797

Angel Rubio: 0000-0003-2060-3151

\section{Present Address}

${ }^{\S}$ Center for Computational Quantum Physics, Flatiron Institute, 162 Fifth Avenue, New York, New York 10010, USA

\section{Notes}

The authors declare no competing financial interest.

\section{ACKNOWLEDGMENTS}

We would like to thank Christian Schäfer and Norah Hoffmann for insightful discussions and Sebastian Ohlmann for the help with the efficient massive parallel implementation. J.F. acknowledges financial support from the Deutsche Forschungsgemeinschaft (DFG) under Contract No. FL 997/1-1, and all of us acknowledge financial support from the European Research Council (ERC-2015-AdG-694097), the Cluster of Excellence 'Advanced Imaging of Matter' (AIM), Grupos Consolidados (IT1249-19) and SFB925 "Light induced dynamics and control of correlated quantum systems". The Flatiron Institute is a division of the Simons Foundation.

\section{REFERENCES}

(1) Ebbesen, T. W. Hybrid Light-Matter States in a Molecular and Material Science Perspective. Acc. Chem. Res. 2016, 49, 2403-2412.

(2) Sukharev, M.; Nitzan, A. Optics of exciton-plasmon nanomaterials. J. Phys.: Condens. Matter 2017, 29, 443003.

(3) George, J.; Shalabney, A.; Hutchison, J. A.; Genet, C.; Ebbesen, T. W. Liquid-Phase Vibrational Strong Coupling. J. Phys. Chem. Lett. 2015, 6, 1027-1031.

(4) Hiura, H.; Shalabney, A.; George, J. Cavity Catalysis Accelerating Reactions under Vibrational Strong Coupling. chemrxiv:7234721 2018, na.

(5) Thomas, A.; Lethuillier-Karl, L.; Nagarajan, K.; Vergauwe, R. M. A.; George, J.; Chervy, T.; Shalabney, A.; Devaux, E.; Genet, C.; Moran, J.; Ebbesen, T. W. Tilting a ground-state reactivity landscape by vibrational strong coupling. Science 2019, 363, 615-619.

(6) Riek, C.; Seletskiy, D. V.; Moskalenko, A. S.; Schmidt, J. F.; Krauspe, P.; Eckart, S.; Eggert, S.; Burkard, G.; Leitenstorfer, A. Direct sampling of electric-field vacuum fluctuations. Science 2015, 350, $420-423$.

(7) Coles, D.; Flatten, L. C.; Sydney, T.; Hounslow, E.; Saikin, S. K.; Aspuru-Guzik, A.; Vedral, V.; Tang, J. K.-H.; Taylor, R. A.; Smith, J. M.; Lidzey, D. G. A Nanophotonic Structure Containing Living Photosynthetic Bacteria. Small 2017, 13, 1701777.

(8) Chikkaraddy, R.; de Nijs, B.; Benz, F.; Barrow, S. J.; Scherman, O. A.; Rosta, E.; Demetriadou, A.; Fox, P.; Hess, O.; Baumberg, J. J. Single-molecule strong coupling at room temperature in plasmonic nanocavities. Nature 2016, 535, 127-130.

(9) Benz, F.; Schmidt, M. K.; Dreismann, A.; Chikkaraddy, R.; Zhang, Y.; Demetriadou, A.; Carnegie, C.; Ohadi, H.; de Nijs, B.; Esteban, R.; Aizpurua, J.; Baumberg, J. J. Single-molecule optomechanics in "picocavities. Science 2016, 354, 726-729.

(10) Kleemann, M.-E.; Chikkaraddy, R.; Alexeev, E.; Kos, D.; Carnegie, C.; Deacon, W.; Casalis De Pury, A.; Grosse, C.; De Nijs, B.; Mertens, J.; Tartakovskii, A.; Baumberg, J. Research data supporting "Strong-coupling of $\mathrm{WSe}_{2}$ in ultra-compact plasmonic nanocavities at room temperature". Nat. Commun. 2017, 8, 1296.

(11) Bisht, A.; Cuadra, J.; Wersäll, M.; Canales, A.; Antosiewicz, T. J.; Shegai, T. Collective Strong Light-Matter Coupling in Hierarchical Microcavity-Plasmon-Exciton Systems. Nano Lett. 2019, 19, 189196.

(12) Mirhosseini, M.; Kim, E.; Zhang, X.; Sipahigil, A.; Dieterle, P. B.; Keller, A. J.; Asenjo-Garcia, A.; Chang, D. E.; Painter, O. Waveguide-mediated interaction of artificial atoms in the strong coupling regime. Nature 2019, 569, 692-697.

(13) Ruggenthaler, M.; Tancogne-Dejean, N.; Flick, J.; Appel, H.; Rubio, A. From a quantum-electrodynamical light-matter description to novel spectroscopies. Nature Reviews Chemistry 2018, 2, 0118.

(14) Flick, J.; Rivera, N.; Narang, P. Strong light-matter coupling in quantum chemistry and quantum photonics. Nanophotonics 2018, 7, $1479-1501$.

(15) Galego, J.; Garcia-Vidal, F. J.; Feist, J. Cavity-Induced Modifications of Molecular Structure in the Strong-Coupling Regime. Phys. Rev. X 2015, 5, 041022.

(16) Galego, J.; Garcia-Vidal, F. J.; Feist, J. Suppressing photochemical reactions with quantized light fields. Nat. Commun. 2016, 7, 13841.

(17) Kowalewski, M.; Bennett, K.; Mukamel, S. Cavity Femtochemistry: Manipulating Nonadiabatic Dynamics at Avoided Crossings. J. Phys. Chem. Lett. 2016, 7, 2050-2054.

(18) Cirio, M.; De Liberato, S.; Lambert, N.; Nori, F. Ground State Electroluminescence. Phys. Rev. Lett. 2016, 116, 113601.

(19) Herrera, F.; Spano, F. C. Cavity-Controlled Chemistry in Molecular Ensembles. Phys. Rev. Lett. 2016, 116, 238301.

(20) Galego, J.; Garcia-Vidal, F. J.; Feist, J. Many-Molecule Reaction Triggered by a Single Photon in Polaritonic Chemistry. Phys. Rev. Lett. 2017, 119, 136001.

(21) Roelli, P.; Galland, C.; Piro, N.; Kippenberg, T. J. Molecular cavity optomechanics as a theory of plasmon-enhanced Raman scattering. Nat. Nanotechnol. 2016, 11, 164-169. 
(22) Shin, D.; Hubener, H.; De Giovannini, U.; Jin, H.; Rubio, A.; Park, N. Phonon-driven spin-Floquet magneto-valleytronics in $\mathrm{MoS}_{2}$. Nat. Commun. 2018, 9, na.

(23) Mazza, G.; Georges, A. Superradiant Quantum Materials. Phys. Rev. Lett. 2019, 122, 017401.

(24) Sentef, M. A.; Ruggenthaler, M.; Rubio, A. Cavity quantumelectrodynamical polaritonically enhanced electron-phonon coupling and its influence on superconductivity. Science Advances 2018, 4, eaau6969.

(25) Braak, D. Integrability of the Rabi Model. Phys. Rev. Lett. 2011, 107, 100401.

(26) Xie, Q.; Zhong, H.; Batchelor, M. T.; Lee, C. The quantum Rabi model: solution and dynamics. J. Phys. A: Math. Theor. 2017, 50, 113001.

(27) Garraway, B. M. The Dicke model in quantum optics: Dicke model revisited. Philos. Trans. R. Soc., A 2011, 369, 1137-1155.

(28) De Bernardis, D.; Pilar, P.; Jaako, T.; De Liberato, S.; Rabl, P. Breakdown of gauge invariance in ultrastrong-coupling cavity QED. Phys. Rev. A: At., Mol., Opt. Phys. 2018, 98, 053819.

(29) Schäfer, C.; Ruggenthaler, M.; Rubio, A. Ab initio nonrelativistic quantum electrodynamics: Bridging quantum chemistry and quantum optics from weak to strong coupling. Phys. Rev. A: At., Mol., Opt. Phys. 2018, 98, 043801.

(30) Gilbert Grynberg, C. F. Alain Aspect. Introduction to Quantum Optics From Semi-classical Approach to Quantized Light; Cambridge University Press: New York, 2010.

(31) Weisskopf, V.; Wigner, E. Berechnung der natürlichen Linienbreite auf Grund der Diracschen Lichttheorie. Eur. Phys. J. A 1930, 63, 54-73.

(32) Bužek, V.; Drobný, G.; Kim, M. G.; Havukainen, M.; Knight, P. L. Numerical simulations of atomic decay in cavities and material media. Phys. Rev. A: At., Mol., Opt. Phys. 1999, 60, 582-592.

(33) Lettow, R.; Ahtee, V.; Pfab, R.; Renn, A.; Ikonen, E.; Götzinger, S.; Sandoghdar, V. Realization of two Fourier-limited solid-state single-photon sources. Opt. Express 2007, 15, 15842-15847.

(34) Wang, D.; Kelkar, H.; Martin-Cano, D.; Utikal, T.; Götzinger, S.; Sandoghdar, V. Coherent Coupling of a Single Molecule to a Scanning Fabry-Perot Microcavity. Phys. Rev. X 2017, 7, 021014.

(35) Ruggenthaler, M. Inducing Multiple Reactions with a Single Photon. Physics 2017, 10, na.

(36) Tokatly, I. V. Time-Dependent Density Functional Theory for Many-Electron Systems Interacting with Cavity Photons. Phys. Rev. Lett. 2013, 110, 233001.

(37) Ruggenthaler, M.; Flick, J.; Pellegrini, C.; Appel, H.; Tokatly, I. V.; Rubio, A. Quantum-electrodynamical density-functional theory: Bridging quantum optics and electronic-structure theory. Phys. Rev. A: At., Mol., Opt. Phys. 2014, 90, 012508.

(38) Ruggenthaler, M. Ground-State Quantum-Electrodynamical Density-Functional Theory. ArXiv e-prints 2015, na.

(39) Flick, J.; Schäfer, C.; Ruggenthaler, M.; Appel, H.; Rubio, A. Ab Initio Optimized Effective Potentials for Real Molecules in Optical Cavities: Photon Contributions to the Molecular Ground State. ACS Photonics 2018, 5, 992-1005.

(40) Hess, O.; Kuhn, T. Maxwell-Bloch equations for spatially inhomogeneous semiconductor lasers. I. Theoretical formulation. Phys. Rev. A: At., Mol., Opt. Phys. 1996, 54, 3347-3359.

(41) Lorin, E.; Chelkowski, S.; Bandrauk, A. A numerical MaxwellSchrödinger model for intense laser-matter interaction and propagation. Comput. Phys. Commun. 2007, 177, 908-932.

(42) Yabana, K.; Sugiyama, T.; Shinohara, Y.; Otobe, T.; Bertsch, G. F. Time-dependent density functional theory for strong electromagnetic fields in crystalline solids. Phys. Rev. B: Condens. Matter Mater. Phys. 2012, 85, 045134.

(43) Ryder, L. H. Quantum Field Theory; Cambridge University Press, 1996

(44) Craig, D.; Thirunamachandran, T. Molecular Quantum Electrodynamics: An Introduction to Radiation-Molecule Interactions; Dover Books on Chemistry Series; Dover Publications, 1998.
(45) Spohn, H. Dynamics of Charged Particles and Their Radiation Field; Cambridge University Press, 2004.

(46) Jestädt, R.; Ruggenthaler, M.; Oliveira, M. J. T.; Rubio, A.; Appel, H. Real-time solutions of coupled Ehrenfest-Maxwell-PauliKohn-Sham equations: fundamentals, implementation, and nanooptical applications. arXiv e-prints, arXiv:1812.05049 2018, na.

(47) Rokaj, V.; Welakuh, D. M.; Ruggenthaler, M.; Rubio, A. Lightmatter interaction in the long-wavelength limit: no ground-state without dipole self-energy. J. Phys. B: At., Mol. Opt. Phys. 2018, 51, 034005 .

(48) Flick, J.; Narang, P. Cavity-Correlated Electron-Nuclear Dynamics from First Principles. Phys. Rev. Lett. 2018, 121, 113002.

(49) Flick, J.; Narang, P. Excited-State Nanophotonic and Polaritonic Chemistry with $\mathrm{Ab}$ initio Potential-Energy Surfaces. arXiv:1907.04646 1907, na.

(50) Pellegrini, C.; Flick, J.; Tokatly, I. V.; Appel, H.; Rubio, A. Optimized Effective Potential for Quantum Electrodynamical TimeDependent Density Functional Theory. Phys. Rev. Lett. 2015, 115, 093001.

(51) Szabo, A.; Ostlund, N. Modern Quantum Chemistry: Introduction to Advanced Electronic Structure Theory; Dover Books on Chemistry; Dover Publications, 1989.

(52) Dimitrov, T.; Flick, J.; Ruggenthaler, M.; Rubio, A. Exact functionals for correlated electron-photon systems. New J. Phys. 2017, $19,113036$.

(53) Hoffmann, N. Response formalism in density-functional theory for quantum electrodynamics. M.Sc. Thesis, Technical University, Berlin, 2016.

(54) Fetter, A. L.; Walecka, J. D. Quantum Theory of Many-Particle Systems; Courier Corporation, 2003.

(55) Stefanucci, G.; van Leeuwen, R. Nonequilibrium Many-Body Theory of Quantum Systems: A Modern Introduction; Cambridge University Press, 2013.

(56) Bonitz, M. Quantum Kinetic Theory; Springer, 1998.

(57) Dreizler, R. M.; Gross, E. K. Density Functional Theory: An Approach to the Quantum Many-Body Problem; Springer Science \& Business Media, 2012.

(58) Engel, E.; Dreizler, R. Density Functional Theory: An Advanced Course; Theoretical and Mathematical Physics; Springer, 2011.

(59) Ullrich, C. A. Time-Dependent Density-Functional Theory: Concepts and Applications; OUP: Oxford, 2011.

(60) Ruggenthaler, M.; van Leeuwen, R. Global fixed-point proof of time-dependent density-functional theory. EPL (Europhysics Letters) 2011, 95, 13001 .

(61) Flick, J.; Ruggenthaler, M.; Appel, H.; Rubio, A. Kohn-Sham approach to quantum electrodynamical density-functional theory: Exact time-dependent effective potentials in real space. Proc. Natl. Acad. Sci. U. S. A. 2015, 112, 15285-15290.

(62) Ruggenthaler, M.; Penz, M.; Van Leeuwen, R. Existence, uniqueness, and construction of the density-potential mapping in time-dependent density-functional theory. J. Phys.: Condens. Matter 2015, 27, 203202.

(63) Maitra, N. T.; Burke, K.; Woodward, C. Memory in TimeDependent Density Functional Theory. Phys. Rev. Lett. 2002, 89, 023002.

(64) Petersilka, M.; Gossmann, U. J.; Gross, E. K. U. Excitation Energies from Time-Dependent Density-Functional Theory. Phys. Rev. Lett. 1996, 76, 1212-1215.

(65) Casida, M. Time-Dependent Density Functional Response Theory of Molecular Systems: Theory, Computational Methods, and Functionals. Recent Developments and Applications of Modern Density Functional Theory; Elsevier Science, 1996.

(66) Ehrenreich, H. Electromagnetic transport in solids: optical properties and plasma effects. The Optical Properties of Solids; Oxford University Press, 1966; p 106.

(67) Mochán, W. L.; Barrera, R. G. Electromagnetic response of systems with spatial fluctuations. I. General formalism. Phys. Rev. B: Condens. Matter Mater. Phys. 1985, 32, 4984. 
(68) Maki, J. J.; Malcuit, M. S.; Sipe, J.; Boyd, R. W. Linear and nonlinear optical measurements of the Lorentz local field. Phys. Rev. Lett. 1991, 67, 972.

(69) Luppi, E.; Hübener, H.; Véniard, V. Ab initio second-order nonlinear optics in solids: Second-harmonic generation spectroscopy from time-dependent density-functional theory. Phys. Rev. B: Condens. Matter Mater. Phys. 2010, 82, 235201.

(70) Rabi, I. I. On the Process of Space Quantization. Phys. Rev. 1936, 49, 324-328.

(71) Rabi, I. I. Space Quantization in a Gyrating Magnetic Field. Phys. Rev. 1937, 51, 652-654.

(72) Shalabney, A.; George, J.; Hutchison, J.; Pupillo, G.; Genet, C.; Ebbesen, T. W. Coherent coupling of molecular resonators with a microcavity mode. Nat. Commun. 2015, 6, 5981.

(73) George, J.; Chervy, T.; Shalabney, A.; Devaux, E.; Hiura, H.; Genet, C.; Ebbesen, T. W. Multiple Rabi Splittings under Ultrastrong Vibrational Coupling. Phys. Rev. Lett. 2016, 117, 153601.

(74) Ott, C.; Kaldun, A.; Raith, P.; Meyer, K.; Laux, M.; Evers, J.; Keitel, C. H.; Greene, C. H.; Pfeifer, T. Lorentz Meets Fano in Spectral Line Shapes: A Universal Phase and Its Laser Control. Science 2013, 340, 716-720.

(75) Castro, A.; Appel, H.; Rubio, A. Optimal control theory for quantum electrodynamics: an initial state problem. The European Physical Journal B 2019, 92 (10), 223.

(76) Marques, M. A.; Castro, A.; Bertsch, G. F.; Rubio, A. octopus: a first-principles tool for excited electron-ion dynamics. Comput. Phys. Commun. 2003, 151, 60-78.

(77) Andrade, X.; et al. Real-space grids and the Octopus code as tools for the development of new simulation approaches for electronic systems. Phys. Chem. Chem. Phys. 2015, 17, 31371-31396.

(78) Yabana, K.; Bertsch, G. F. Time-dependent local-density approximation in real time: Application to conjugated molecules. Int. J. Quantum Chem. 1999, 75, 55-66.

(79) Pantos, E.; Philis, J.; Bolovinos, A. The extinction coefficient of benzene vapor in the region 4.6 to $36 \mathrm{eV}$. J. Mol. Spectrosc. 1978, 72, 36-43.

(80) Dawes, A.; Pascual, N.; Hoffmann, S. V.; Jones, N. C.; Mason, N. J. Vacuum ultraviolet photoabsorption spectroscopy of crystalline and amorphous benzene. Phys. Chem. Chem. Phys. 2017, 19, 2754427555.

(81) Coles, D. M.; Yang, Y.; Wang, Y.; Grant, R. T.; Taylor, R. A.; Saikin, S. K.; Aspuru-Guzik, A.; Lidzey, D. G.; Tang, J. K.-H.; Smith, J. M. Strong coupling between chlorosomes of photosynthetic bacteria and a confined optical cavity mode. Nat. Commun. 2014, 5, 5561.

(82) George, J.; Wang, S.; Chervy, T.; Canaguier-Durand, A.; Schaeffer, G.; Lehn, J.-M.; Hutchison, J. A.; Genet, C.; Ebbesen, T. W. Ultra-strong coupling of molecular materials: spectroscopy and dynamics. Faraday Discuss. 2015, 178, 281-294.

(83) Flick, J.; Ruggenthaler, M.; Appel, H.; Rubio, A. Atoms and molecules in cavities, from weak to strong coupling in quantumelectrodynamics (QED) chemistry. Proc. Natl. Acad. Sci. U. S. A. 2017, 114, 3026-3034.

(84) Milonni, P. Semiclassical and quantum-electrodynamical approaches in nonrelativistic radiation theory. Phys. Rep. 1976, 25, $1-81$.

(85) Di Piazza, A.; Müller, C.; Hatsagortsyan, K.; Keitel, C. Extremely high-intensity laser interactions with fundamental quantum systems. Rev. Mod. Phys. 2012, 84, 1177.

(86) Firstenberg, O.; Peyronel, T.; Liang, Q.-Y.; Gorshkov, A. V.; Lukin, M. D.; Vuletić, V. Attractive photons in a quantum nonlinear medium. Nature 2013, 502, 71-75.

(87) Dorfman, K. E.; Schlawin, F.; Mukamel, S. Nonlinear optical signals and spectroscopy with quantum light. Rev. Mod. Phys. 2016, 88,045008 .

(88) Blanchard, P.; Brüning, E. Mathematical Methods in Physics: Distributions, Hilbert Space Operators, Variational Methods, and Applications in Quantum Physics; Birkhäuser, 2015; Vol. 69.

(89) Teschl, G. Mathematical Methods in Quantum Mechanics; American Mathematical Soc., 2014; Vol. 157.
(90) Bauernschmitt, R.; Ahlrichs, R. Treatment of electronic excitations within the adiabatic approximation of time dependent density functional theory. Chem. Phys. Lett. 1996, 256, 454-464.

(91) Jaynes, E.; Cummings, F. Comparison of quantum and semiclassical radiation theories with application to the beam maser. Proc. IEEE 1963, 51, 89-109.

(92) Kohn, W.; Sham, L. J. Self-Consistent Equations Including Exchange and Correlation Effects. Phys. Rev. 1965, 140, A1133A1138.

(93) Perdew, J. P.; Zunger, A. Self-interaction correction to densityfunctional approximations for many-electron systems. Phys. Rev. B: Condens. Matter Mater. Phys. 1981, 23, 5048-5079.

(94) Troullier, N.; Martins, J. L. Efficient pseudopotentials for planewave calculations. Phys. Rev. B: Condens. Matter Mater. Phys. 1991, 43, 1993-2006. 